October, 2003

preprint UT 03-35

hep-th/0310235

\title{
Topological Strings and Nekrasov's formulas
}

\author{
Tohru Eguchi \\ Department of Physics, Faculty of Science \\ University of Tokyo, Tokyo, 113-0033, Japan \\ Hiroaki Kanno \\ Graduate School of Mathematics \\ Nagoya University, Nagoya, 464-8602, Japan
}

\begin{abstract}
We apply the method of geometric transition and compute all genus topological closed string amplitudes compactified on local $\mathbf{F}_{0}$ by making use of the ChernSimons gauge theory. We find an exact agreement of the results of our computation with the formula proposed recently by Nekrasov for $\mathcal{N}=2 S U(2)$ gauge theory with two parameters $\beta$ and $\hbar$. $\beta$ is related to the size of the fiber of $\mathbf{F}_{0}$ and $\hbar$ corresponds to the string coupling constant.

Thus Nekrasov's formula encodes all the information of topological string amplitudes on local $\mathbf{F}_{0}$ including the number of holomorphic curves at arbitrary genus. By taking suitable limits $\beta$ and/or $\hbar \rightarrow 0$ one recovers the four-dimensional SeibergWitten theory and also its coupling to external graviphoton fields.

We also compute topological string amplitude for the local 2nd del Pezzo surface and check the consistency with Nekrasov's formula of $S U(2)$ gauge theory with a matter field in the vector representation.
\end{abstract}




\section{Introduction}

Recently the method of geometric transition has been applied to various cases of local Calabi-Yau manifolds $M$ and the topological amplitudes of closed string theory compactified on $M$ have been computed using the Chern-Simons theory [1, 2, 3, 4, 15, 6]. In particular the cases of Calabi-Yau manifolds which are canonical bundles $K_{S}$ over rational surfaces $S$ such as $\mathbf{P}^{2}$, Hirzebruch surfaces $\mathbf{F}_{n}$ and also del Pezzo surfaces have been studied in detail. It has been shown that the structure of Gopakumar and Vafa [7] for the number of higher genus holomorphic curves emerges automatically from the all genus Chern-Simons calculations and currently the method of geometric transition is the most efficient way of computing higher genus amplitudes of topological strings.

On the other hand, using the technique of localization theory Nekrasov [8] has recently proposed a formula for the instanton amplitudes of $\mathcal{N}=2$ SUSY gauge theory at arbitrary instanton numbers: his formula contains two parameters $\beta$ and $\hbar$ and involves the sum over a pair of Young diagrams in the case of $S U(2)$ gauge group. In the limit $\beta, \hbar \rightarrow 0$ Nekrasov's expression reduces to the well-known results of Seiberg and Witten of four-dimensional gauge theory [9]. See also [10, 11, 12] for recent studies on Nekrasov's formula.

In 4] Iqbal and Kashani-Poor used the method of geometric transition for the local $\mathbf{F}_{0}$ and reproduced Nekrasov's expression by postulating some identities involving Hopf-link invariants in Chern-Simons theory. They were, however, mainly concerned with the case of field theory limit $\beta, \hbar \rightarrow 0$.

In this paper we prove these identities and compute rigorously the all genus topological string amplitudes on local $\mathbf{F}_{0}$. We find an exact agreement of our results with the formula of Nekrasov with parameters $\beta, \hbar$ kept being non-zero: these parameters match with the corresponding ones of Chern-Simons theory. We note that when the parameter $\beta$ is identified as the radius $R$ of the fifth-dimensional circle, Nekrasov's expression exactly reproduces the instanton amplitudes of five-dimensional gauge theory on $\mathbf{R}^{4} \times S^{1}$ at $\hbar=0$. We recall that instanton amplitudes in five-dimensional gauge theories count the 
number of genus zero holomorphic curves in Calabi-Yau manifolds [13, 14, 15, 16].

Agreement with the all genus amplitudes obtained by the Chern-Simons calculation implies that the higher order terms in $\hbar$ of Nekrasov's formula count precisely the number of higher genus holomorphic curves. Therefore Nekrasov's expression encodes all the information of closed topological string amplitudes compactified on the local Calabi-Yau manifold $K_{\mathbf{F}_{0}}$.

If one takes the four-dimensional limit $\beta \rightarrow 0$ with $\hbar$ kept finite, one obtains the amplitudes of the four-dimensional $\mathcal{N}=2$ gauge theory in the graviphoton background as suggested by 8 .

In the following we will provide proofs for some of the identities involving the Hopflink invariants postulated in previous papers. We also study the local del Pezzo surfaces which are obtained by blowing up local Hirzebruch surfaces and describe gauge theories with additional matter hypermultiplets. We compute the topological string amplitude for the 2nd del Pezzo surface and recover Nekrasov's expression of $S U(2)$ gauge theory in the presence of a matter in the vector representation.

\section{Toric diagram and topological string amplitudes}

In the following we will consider a class of toric rational surfaces $S$ that includes $\mathbf{P}^{2}, \mathbf{F}_{0}=$ $\mathbf{P}^{1} \times \mathbf{P}^{1}, \mathbf{F}_{2}$ and their blow ups. The canonical line bundle $K_{S}$ over $S$ is a non-compact Calabi-Yau manifold. We will compute topological closed string amplitudes compactified on the manifolds $K_{S}$. In general toric diagrams describe patterns of degeneration of the torus action. For a toric rational surface $S$ with $\mathbf{T}^{2}$ action, the diagram is made of a polygon with $N$ vertices and an external line attached to each trivalent vertex. Along the edges of the polygon an $S^{1}$ action specified by the direction of each edge degenerates and at the vertices both of the $\mathbf{T}^{2}$ actions become degenerate. The diagram looks like a one loop Feynman graph with $N$ external lines, where $N=3$ for $\mathbf{P}^{2}, N=4$ for $\mathbf{F}_{m}$ and blowing up at a point increases $N$ by one. We take the clockwise direction in 
the loop and assign to the $i$-th internal edge a representation $R_{i}(i=1, \cdots, N)$ and the (renormalized) Kähler parameter $t_{i}$. Note that by the way of degeneration of toric action explained above, we have a rational curve for each internal edge. All external lines have the trivial representation that will be denoted by $\bullet$. Throughout the paper we will identify representation $R$ with its Young diagram $\mu^{R}$, specified by the number of boxes $\mu_{j}$ in the $j$-th row. The $\mu_{j}$ 's are non-increasing; $\mu_{1} \geq \mu_{2} \geq \cdots \geq \mu_{d}>\mu_{d+1}=0$, where $d:=d\left(\mu^{R}\right)$ is the "depth" i.e. the number of rows of the diagram. We denote the total number of boxes by $\ell_{R}$;

$$
\ell_{R}:=\sum_{j=1}^{d} \mu_{j}
$$

The Young diagram represents a partition of $\ell_{R}$ objects. The integer $\kappa_{R}$ defined by

$$
\kappa_{R}:=2 \sum_{j=1}^{d} \sum_{k=1}^{\mu_{j}}(k-j)=\ell_{R}+\sum_{j=1}^{d} \mu_{j}\left(\mu_{j}-2 j\right)
$$

plays also an important role in the following. It is related to the quadratic Casimir of the representation $R$ of the group $U(N)$ by

$$
C_{R}=\kappa_{R}+N \ell_{R}
$$
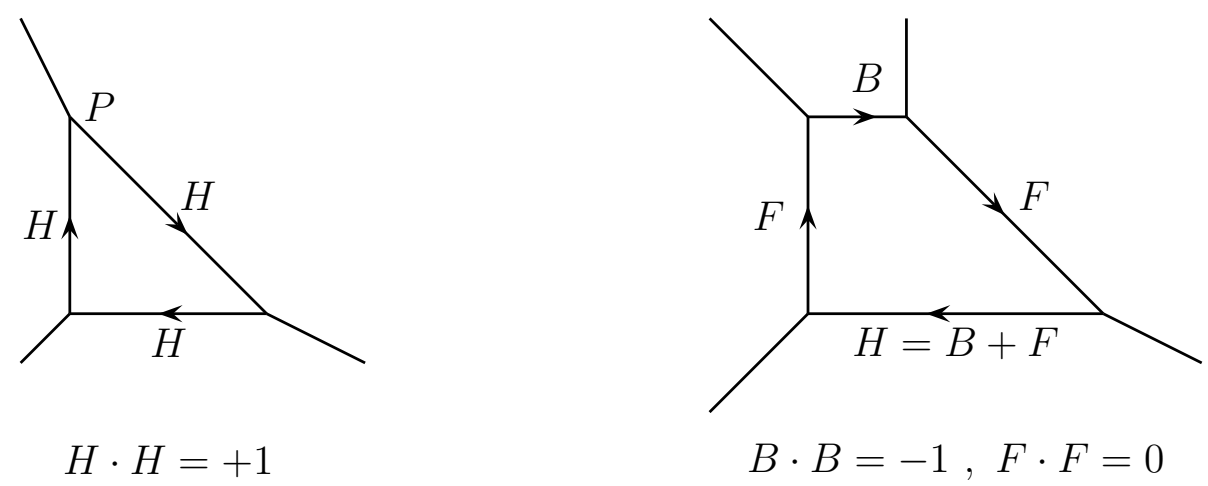

Figure 1 : Toric diagram of $\mathbf{P}^{2}$ (left) and its blow up $\mathbf{F}_{1}$ at $\mathrm{P}$ (right) 
Based on the rules proposed by Iqbal [3] and the idea of topological vertex [17], we find the following universal formula for topological closed string amplitude compactified on local Calabi-Yau manifolds $K_{S}$;

$$
\begin{aligned}
Z_{\text {top str }}^{(S)}= & \sum_{R_{1} \cdots R_{N}} W_{R_{N} R_{1}} W_{R_{1} R_{2}} \cdots W_{R_{N-1} R_{N}} \cdot e^{-\sum_{i=1}^{N} t_{i} \cdot \ell_{R_{i}}} \\
& \times(-1)^{\sum_{i=1}^{N} \gamma_{i} \cdot \ell_{R_{i}}} q^{\frac{1}{2} \sum_{i=1}^{N} \gamma_{i} \cdot \kappa_{R_{i}}}
\end{aligned}
$$

where $q=\exp (2 \pi i /(N+k))$ and $W_{R_{i} R_{j}}$ is the Chern-Simons invariant of the Hopf link (with the standard framing) in $S^{3}$ carrying representations $R_{i}$ and $R_{j}$ (see Appendix A). The "propagator" for the $i$-th internal line is given by $e^{-t_{i} \cdot \ell_{R_{i}}}$. The last two factors come from the choice of the framing. It turns out that we can fix the framing by the self-intersection number $\gamma_{i}$ of the rational curve associated with the $i$-th edge. In deriving the above formula we have used the following relation to the topological vertex $C_{R_{1}, R_{2}, R_{3}}$ introduced in [17]

$$
C_{\bullet, R_{2}, R_{3}^{t}}=W_{R_{2} R_{3}} q^{-\frac{1}{2} \kappa_{R_{3}}}
$$

where $R^{t}$ denotes the conjugate representation obtained by exchanging row and columns of the Young diagram. According to [17] the topological vertex has the cyclic symmetry;

$$
C_{R_{1}, R_{2}, R_{3}}=C_{R_{2}, R_{3}, R_{1}}=C_{R_{3}, R_{1}, R_{2}}
$$

and the conjugation property;

$$
C_{R_{1}, R_{2}, R_{3}}=q^{\frac{1}{2} \sum_{i} \kappa_{R_{i}}} C_{R_{1}^{t}, R_{3}^{t}, R_{2}^{t}} .
$$

In particular the last property implies;

$$
W_{R_{1} R_{2}}=q^{\frac{1}{2} \kappa_{R_{2}}} C_{\bullet, R_{1}, R_{2}^{t}}=q^{\frac{1}{2} \kappa_{R_{1}}} C_{\bullet, R_{2}, R_{1}^{t}}=W_{R_{2} R_{1}}
$$

where we have used $\kappa_{R^{t}}=-\kappa_{R}$. Let us consider $\mathbf{P}^{2}$ as an example. The toric diagram is a triangle $(N=3)$ and all the edges represent the hyperplane class $H$ of $\mathbf{P}^{2}$ with the self-intersection $H \cdot H=1$. Thus our universal formula implies

$$
\begin{aligned}
Z_{\text {top str }}^{\left(\mathbf{P}^{2}\right)}= & \sum_{R_{1}, R_{2}, R_{3}} W_{R_{1} R_{2}} W_{R_{2} R_{3}} W_{R_{3} R_{1}} \cdot e^{-t_{H} \cdot\left(\ell_{R_{1}}+\ell_{R_{2}}+\ell_{R_{3}}\right)} \\
& \times(-1)^{\ell_{R_{1}}+\ell_{R_{2}}+\ell_{R_{3}}} q^{\frac{1}{2}\left(\kappa_{R_{1}}+\kappa_{R_{2}}+\kappa_{R_{3}}\right)}
\end{aligned}
$$


where $t_{H}$ is the Kähler moduli for $H$. This topological string amplitude has been extensively investigated in [1].

\section{Local Hirzebruch surface and five dimensional gauge theory}

The Hirzebruch surface $\mathbf{F}_{m}$ is a $\mathbf{P}^{1}$ bundle over $\mathbf{P}^{1}$. The second homology class $H_{2}\left(\mathbf{F}_{m}, \mathbf{Z}\right)$ is spanned by the two cycles $B$ and $F$, where their representatives are the base $\mathbf{P}^{1}$ and the $\mathbf{P}^{1}$ fiber, respectively. The intersection numbers of these cycles are

$$
B \cdot B=-m, \quad F \cdot F=0, \quad B \cdot F=+1 .
$$

The Hirzebruch surfaces $\mathbf{F}_{m}(m=0,1,2)$ have been used in geometric engineering of $\mathcal{N}=2$ pure Yang-Mills theory with $S U(2)$ gauge symmetry [18, 19, 20].



$B \cdot B=0, F \cdot F=0$

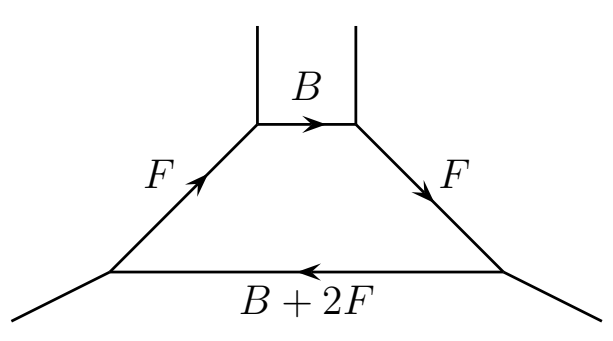

$B \cdot B=-2, F \cdot F=0$

Figure 2 : Toric diagram of the Hirzebruch surfaces $\mathbf{F}_{0}$ (left) and $\mathbf{F}_{2}$ (right)

From the toric diagram of $\mathbf{F}_{m}(m=0,1,2)$ and the data of self-intersection numbers we can write down all genus topological string amplitude;

$$
\begin{aligned}
Z_{\text {top str }}^{\left(\mathbf{F}_{m}\right)}= & \sum_{R_{1} \cdots R_{4}} W_{R_{4} R_{1}} W_{R_{1} R_{2}} W_{R_{2} R_{3}} W_{R_{3} R_{4}} \cdot e^{-t_{F} \cdot\left(\ell_{R_{1}}+\ell_{R_{3}}+m \ell_{R_{4}}\right)-t_{B} \cdot\left(\ell_{R_{2}}+\ell_{R_{4}}\right)} \\
& \times(-1)^{m\left(\ell_{R_{4}}-\ell_{R_{2}}\right)} q^{\frac{m}{2}\left(\kappa_{R_{4}}-\kappa_{R_{2}}\right)}
\end{aligned}
$$


where $t_{B}$ and $t_{F}$ are the (renormalized) Kähler parameters of $B$ and $F$. Following [4, we introduce the function

$$
K_{R_{1} R_{2}}(Q):=\sum_{S} Q^{\ell_{S}} W_{R_{1} S}(q) W_{S R_{2}}(q) .
$$

The amplitude can be written as

$$
Z_{\text {top str }}^{\left(\mathbf{F}_{m}\right)}=\sum_{R_{1} R_{2}}\left(K_{R_{1} R_{2}}\left(Q_{F}\right)\right)^{2} \cdot Q_{B}^{\ell_{R_{1}}+\ell_{R_{2}}} Q_{F}^{m \ell_{R_{2}}} \cdot(-1)^{m\left(\ell_{R_{2}}-\ell_{R_{1}}\right)} q^{\frac{m}{2}\left(\kappa_{R_{2}}-\kappa_{R_{1}}\right)},
$$

where $Q_{B}:=e^{-t_{B}}$ and $Q_{F}:=e^{-t_{F}}$. Assuming that the contributions are exhausted by multiple-covers of isolated rational curves, Iqbal and Kashani-Poor have proposed the following proposition;

\section{Proposition 1}

$$
K_{R_{1} R_{2}}(Q)=W_{R_{1}}(q) W_{R_{2}}(q) \exp \left(\sum_{n=1}^{\infty} \frac{\widetilde{f}_{R_{1} R_{2}}\left(q^{n}\right)}{n} Q^{n}\right) .
$$

Here the function $\widetilde{f}_{R_{1} R_{2}}$ is given by

$$
\begin{aligned}
& \widetilde{f}_{R_{1} R_{2}}(q):=W_{\square}^{2}(q)+f_{R_{1}}(q)+f_{R_{2}}(q)+f_{R_{1}}(q) f_{R_{2}}(q) W_{\square}^{-2}(q), \\
& f_{R}(q):=\sum_{i=1}^{d} \sum_{k=1}^{\mu_{i}} q^{k-i}=\frac{q}{(q-1)} \sum_{i=1}^{d}\left(q^{\mu_{i}-i}-q^{-i}\right), \\
& W_{\square}(q):=\frac{1}{[1]}=\frac{q^{\frac{1}{2}}}{q-1} .
\end{aligned}
$$

We now present a (somewhat lengthy) proof of the important identity (3.5): we start from the formula of the Hopf link invariants (see Appendix A);

$$
W_{R_{1} R_{2}}(q)=W_{R_{1}}(q) q^{\frac{\ell_{R_{2}}}{2}} s_{\mu^{R_{2}}}\left(E_{\mu^{R_{1}}}\right) \text {. }
$$

Here $s_{\mu^{R_{2}}}$ denotes the Schur function for the representation $R_{2}$ defined by the JacobiTrudy formula

$$
s_{\mu^{R_{2}}}=\operatorname{det}\left(e_{i}^{\mu_{i}^{\prime}-i+j}\right),
$$

where $\mu^{\prime}=\mu^{R_{2}{ }^{t}}$ denotes the $\mu$-parameters of the dual Young diagram. $\left\{e_{i}\right\}$ are elementary symmetric polynomials of the basic variables $x_{i}$

$$
e_{i}=\sum_{j_{1}<j_{2}<\cdots<j_{i}} x_{j_{1}} x_{j_{2}} \cdots x_{j_{i}} .
$$


In terms of $\left\{x_{i}\right\}$ the Schur function is given by

$$
s_{\mu^{R}}\left(x_{i}\right)=\frac{\operatorname{det}\left(x_{i}^{\mu_{j}+n-j}\right)}{\operatorname{det}\left(x_{i}^{n-j}\right)} \text {. }
$$

In evaluating (3.9) we substitute the values $e_{i}^{R_{1}}$ defined by the expansion

$$
E_{\mu^{R_{1}}}(t)=\sum_{i=0}^{\infty} e_{i}^{R_{1}}(q) t^{i},
$$

into $e_{i}$. We will see that $e_{i}$ defined by this expansion in fact agrees with the expression (3.11) when we suitably specialize the values of $x_{i}$.

From the definition (A.6) of $E_{\mu^{R}}(t)$, one easily finds

$$
e_{1}^{R}(q)=\frac{(q-1)}{q} \widetilde{f}_{R}(q)
$$

where

$$
\widetilde{f}_{R}(q):=f_{R}(q)+W_{\square}^{2}(q)
$$

We note the factorization property of $\widetilde{f}_{R_{1} R_{2}}(q)$

$$
\tilde{f}_{R_{1} R_{2}}(q)=W_{\square}^{-2}(q) \tilde{f}_{R_{1}}(q) \tilde{f}_{R_{2}}(q)=q e_{1}^{R_{1}}(q) e_{1}^{R_{2}}(q)
$$

Next let us introduce the character $\chi^{(\mu)}$ of a representation $\mu$ of the symmetric group and let $\chi_{(\vec{j})}^{(\mu)}$ be its value on the conjugacy class $(\vec{j})=\left(1^{n_{1}}, 2^{n_{2}}, \cdot \cdot, j^{n_{j}}, \cdots\right)$. The Frobenius formula for the character is given by

$$
\prod_{j}\left(p_{j}\left(x_{i}\right)\right)^{n_{j}} \operatorname{det}\left(x_{i}^{n-k}\right)=\sum_{\mu} \chi_{(\vec{j})}^{(\mu)} \operatorname{det}\left(x_{i}^{\mu_{k}+n-k}\right)
$$

where

$$
p_{j}\left(x_{i}\right):=\sum_{i} x_{i}{ }^{j}
$$

is the power sum. (3.17) may be rewritten as

$$
\sum_{\mu} \chi_{(\vec{j})}^{(\mu)} s_{\mu}\left(x_{i}\right)=\prod_{j}\left(p_{j}\left(x_{i}\right)\right)^{n_{j}} .
$$


We may identify the right-hand-side of (3.19) as Schur functions in the winding basis

$$
s_{\vec{j}}\left(x_{i}\right)=\prod_{j}\left(p_{j}\left(x_{i}\right)\right)^{n_{j}} .
$$

Thus in the winding basis Schur functions become monomials in the power sum. Now from (3.14) we find the following relation

$$
\begin{aligned}
& e_{1}^{R}(q)=\sum_{i=1}^{d}\left(q^{\mu_{i}-i}-q^{-i}\right)+\frac{1}{(q-1)} \\
& =\sum_{i=1}^{\infty}\left(q^{\mu_{i}-i}-q^{-i}\right)+\frac{1}{(q-1)}=\sum_{i=1}^{\infty} q^{\mu_{i}-i} .
\end{aligned}
$$

Thus the function $e_{1}^{R}(q)$ is given by the sum of variables $x_{i}$ specialized at

$$
x_{i}=q^{\mu_{i}-i}, \quad i=1,2,3, \cdots .
$$

General power sum is similarly given by

$$
p_{j}\left(x_{i}=q^{\mu_{i}-i}\right)=e_{1}^{R}\left(q^{j}\right) .
$$

Note that if we use the standard relation between the generating functions of $e_{j}\left(x_{i}\right)$ and $p_{j}\left(x_{i}\right)$, we find

$$
\sum_{j=0}^{\infty} e_{j}\left(x_{i}\right) t^{j}=\exp \left(\sum_{j=1}^{\infty} \frac{(-1)^{j+1}}{j} p_{j}\left(x_{i}\right) t^{j}\right)=\exp \left(\sum_{j=1}^{\infty} \frac{(-1)^{j+1}}{j} e_{1}\left(q^{j}\right) t^{j}\right) .
$$

Thus $e_{j}(q)$ for any $j$ can be expressed in terms of $e_{1}\left(q^{i}\right),(i=1, \cdots, j)$.

Now by using (3.23) we find

$$
s_{\vec{j}}\left(x_{i}\right)=\prod_{j}\left(e_{1}\left(q^{j}\right)\right)^{n_{j}} .
$$

By evaluating $K_{R_{1} R_{2}}(q)$ in the winding-basis and using (3.25) we obtain

$$
\begin{aligned}
K_{R_{1} R_{2}}(q) & =W_{R_{1}}(q) W_{R_{2}}(q) \sum_{n_{j}} \prod_{j} \frac{1}{j^{n_{j}} n_{j} !} \prod_{j}\left(e_{1}^{R_{1}}\left(q^{j}\right)\right)^{n_{j}} \prod_{j}\left(e_{1}^{R_{2}}\left(q^{j}\right)\right)^{n_{j}}(q Q)^{\sum j n_{j}} \\
& =W_{R_{1}}(q) W_{R_{2}}(q) \exp \left(\sum_{n} \frac{q^{n}}{n} e_{1}^{R_{1}}\left(q^{n}\right) e_{1}^{R_{2}}\left(q^{n}\right) Q^{n}\right) .
\end{aligned}
$$


The normalization factor $\prod 1 / j^{n_{j}} n_{j}$ ! comes from the orthogonality relation of the characters

$$
\sum_{\vec{j}} \frac{1}{\prod_{j} n_{j} ! j^{n_{j}}} \chi_{(\vec{j})}^{\left(\mu^{R}\right)} \cdot \chi_{(\vec{j})}^{\left(\mu^{S}\right)}=\delta_{R S}
$$

This is the formula we wanted to prove.

If we set $R_{1}=R_{2}=\bullet$ in the above formula, we recover the postulated identity for evaluating the Chern-Simons amplitude in the deformed conifold [3];

$$
\sum_{R}\left(\operatorname{dim}_{q} R\right)^{2} Q^{\ell_{R}}=\exp \left(\sum_{j} \frac{1}{j} \frac{q^{j}}{\left(q^{j}-1\right)^{2}} Q^{j}\right) .
$$

In the following discussions it is convenient to introduce the expansion coefficients $C_{k}\left(R_{1}, R_{2}\right)$ by

$$
f_{R_{1} R_{2}}(q)=f_{R_{1}}(q)+f_{R_{2}}(q)+\left(q+q^{-1}-2\right) f_{R_{1}}(q) f_{R_{2}}(q)=\sum_{k} C_{k}\left(R_{1}, R_{2}\right) q^{k}
$$

Then we have

$$
K_{R_{1} R_{2}}(Q)=W_{R_{1}}(q) W_{R_{2}}(q) \exp \left(\sum_{n=1}^{\infty} \frac{W_{\square}^{2}\left(q^{n}\right)}{n} Q^{n}\right) \prod_{k}\left(1-q^{k} Q_{F}\right)^{-C_{k}\left(R_{1}, R_{2}\right)} .
$$

\subsection{Nekrasov's conjecture for five dimensional gauge theory}

We now would like to show the equality of Nekrasov's formula for five dimensional gauge theory on $\mathbf{R}^{4} \times S^{1}$ and all genus topological string amplitude for local toric Calabi-Yau manifold. More precisely, we prove that the instanton expansion of the partition function of the pure $S U(2)$ Yang-Mills theory on $\mathbf{R}^{4} \times S^{1}$ is exactly the same as the expansion of all genus topological string amplitude for local Hirzebruch surface $\mathbf{F}_{0}=\mathbf{P}^{1} \times \mathbf{P}^{1}$, where the expansion parameter of topological string is an appropriate combination of the Kähler parameters of $\mathbf{F}_{0}$. Due to the presence of the framing factor coming from non-trivial self-intersection of the base $B$ and the fiber $F$, the surfaces $\mathbf{F}_{1}$ and $\mathbf{F}_{2}$ give different amplitudes as five dimensional gauge theory. But they have the same four dimensional limit as $\mathbf{F}_{0}$. 
According to Nekrasov we introduce a pair of representations $R_{1}, R_{2}$ (of $U(N)$ ) in the case of $S U(2)$ gauge theory. The Young tableaux $\mu^{R_{\ell}}$ of the representation $R_{\ell}$ gives a sequence of non-increasing integers; $\mu_{\ell, 1} \geq \mu_{\ell, 2} \geq \cdots \geq \mu_{\ell, d\left(\mu^{R_{\ell}}\right)}>\mu_{\ell, d\left(\mu^{\left.R_{\ell}\right)+1}\right.}=0$, where $\mu_{\ell, i}$ is the number of boxes in the $i$-th row of the Young tableaux $\mu^{R_{\ell}}$ and $d\left(\mu^{R_{\ell}}\right)$ is the depth of $\mu^{R_{\ell}}$. Then Nekrasov's conjecture for the $k$-instanton contribution to the partition function is given by

$$
Z_{k}^{(5 D)}=\sum_{\ell_{R_{1}}+\ell_{R_{2}}=k,} \prod_{\ell \in\{1,2\}} \prod_{i, j=1}^{\infty} \frac{\sinh R\left(a_{\ell m}+\hbar\left(\mu_{\ell, i}-\mu_{m, j}+j-i\right)\right)}{\sinh R\left(a_{\ell m}+\hbar(j-i)\right)}
$$

where $a_{\ell, m}=a_{\ell}-a_{m}$ and we have identified the parameter $\beta$ with the radius $R$ of the circle. For $S U(2)$ gauge theory we have $a_{12}=-a_{21}=2 a$. The partition function is decomposed into two factors; the factor $Z_{k}^{(5 D, 1)}$ with $\ell=m$ and $Z_{k}^{(5 D, 2)}$ with $\ell \neq m$. As topological string amplitude they have different origins as we shall see. Each factor can be simplified as follows (we suppress the restriction $\ell_{R_{1}}+\ell_{R_{2}}=k$ );

$$
\begin{gathered}
Z_{k}^{(5 D, 1)}=\prod_{\ell=1,2} \prod_{i \neq j}^{\infty} \frac{\sinh R \hbar\left(\mu_{\ell, i}-\mu_{\ell, j}+j-i\right)}{\sinh R \hbar(j-i)} \\
=\prod_{\ell=1,2} \prod_{1 \leq i<j<\infty} \frac{\sinh ^{2} R \hbar\left(\mu_{\ell, i}-\mu_{\ell, j}+j-i\right)}{\sinh ^{2} R \hbar(j-i)} \\
Z_{k}^{(5 D, 2)}=\prod_{i, j=1}^{\infty} \frac{\sinh R\left(2 a+\hbar\left(\mu_{1, i}-\mu_{2, j}+j-i\right)\right)}{\sinh R(2 a+\hbar(j-i))} \frac{\sinh R\left(-2 a+\hbar\left(\mu_{2, i}-\mu_{1, j}+j-i\right)\right)}{\sinh R(-2 a+\hbar(j-i))} \\
=\prod_{i, j=1}^{\infty} \frac{\sinh ^{2} R\left(2 a+\hbar\left(\mu_{1, i}-\mu_{2, j}+j-i\right)\right)}{\sinh ^{2} R(2 a+\hbar(j-i))}
\end{gathered}
$$

As we have shown before, all genus topological string amplitude for the local Hirzebruch surface $\mathbf{F}_{m}(m=0,1,2)$ is given by

$$
\begin{aligned}
Z_{\text {top str }}^{\left(\mathbf{F}_{m}\right)}=\exp & \left(\sum_{n=1}^{\infty} \frac{2}{n} W_{\square}^{2}\left(q^{n}\right) Q_{F}^{n}\right) \sum_{R_{1}, R_{2}} Q_{B}^{\ell_{R_{1}}+\ell_{R_{2}}} Q_{F}^{m \ell_{R_{2}}}(-1)^{m\left(\ell_{R_{2}}-\ell_{R_{1}}\right)} q^{-\frac{m}{2}\left(\kappa_{R_{1}}+\kappa_{R_{2}}\right)} \\
& \times W_{R_{1}}^{2}(q) W_{R_{2}^{t}}^{2}(q) \prod_{k}\left(1-q^{k} Q_{F}\right)^{-2 C_{k}\left(R_{1}, R_{2}^{t}\right)}
\end{aligned}
$$


where we have made a replacement $R_{2} \rightarrow R_{2}^{t}$, which is immaterial since we sum over the representations $R_{2}$. The factor $W_{R}(q)$ is the quantum dimension of the representation $R$ or the knot invariant for an unknot in $S^{3}$ with the representation $R$;

$$
\begin{aligned}
W_{R}(q)=\operatorname{dim}_{q} R & =q^{\frac{\kappa_{R}}{4}} \prod_{1 \leq i<j \leq d} \frac{\left[\mu_{i}-\mu_{j}+j-i\right]}{[j-i]} \prod_{i=1}^{d} \prod_{k=1}^{\mu_{i}} \frac{1}{[k-i+d]} \\
& =q^{\frac{\kappa_{R}}{4}} \prod_{1 \leq i<j<\infty} \frac{\left[\mu_{i}-\mu_{j}+j-i\right]}{[j-i]}
\end{aligned}
$$

We note the relation

$$
W_{R^{t}}(q)=q^{-\frac{\kappa_{R}}{2}} W_{R}(q)
$$

The first factor in (3.34) gives the perturbative one-loop contribution to the prepotential. The $k$-instanton part is identified as the sum of terms obeying the condition $\ell_{R_{1}}+\ell_{R_{2}}=k$ as in the Nekrasov's formula. The framing factor depends on the self-intersection numbers of the divisors in $\mathbf{F}_{m}$ and it becomes trivial for $\mathbf{F}_{0}$. We identify the parameters of the gauge theory side as

$$
q=e^{-2 R \hbar}, \quad Q_{F}=e^{-4 R a} .
$$

Then using the identities

$$
\sum_{k} C_{k}\left(R_{1}, R_{2}\right)=\ell_{R_{1}}+\ell_{R_{2}}, \quad \sum_{k} k C_{k}\left(R_{1}, R_{2}\right)=\frac{1}{2}\left(\kappa_{R_{1}}+\kappa_{R_{2}}\right)
$$

we obtain

$$
\prod_{k}\left(1-q^{k} Q_{F}\right)^{-2 C_{k}\left(R_{1}, R_{2}^{t}\right)}=\left(4 Q_{F}\right)^{-\ell_{R_{1}}-\ell_{R_{2}}} q^{-\frac{1}{2}\left(\kappa_{R_{1}}-\kappa_{R_{2}}\right)} \prod_{k} \frac{1}{[\sinh R(2 a+\hbar k)]^{2 C_{k}\left(R_{1}, R_{2}^{t}\right)}}
$$

where $\kappa_{R}=-\kappa_{R^{t}}$.

Establishing the equality $Z_{k}^{(5 D)}$ and $Z_{\text {top str,k }}^{\left(\mathbf{F}_{m}\right)}$ then boils down to the proof of the following proposition;

\section{Proposition 2}

$$
\prod_{k} \frac{1}{\sinh R(2 a+\hbar k)^{C_{k}\left(R_{1}, R_{2}^{t}\right)}}=\prod_{i, j=1}^{\infty} \frac{\sinh R\left(2 a+\hbar\left(\mu_{1, i}-\mu_{2, j}+j-i\right)\right)}{\sinh R(2 a+\hbar(j-i))}
$$


This identity has been postulated by Iqbal and Kashani-Poor. We present a proof in the Appendix B.

The $\kappa$ factors arising in the calculation cancel completely in the case of $\mathbf{F}_{0}$ we find a complete agreement between Nekrasov's expression and all genus topological string amplitude on $\mathbf{F}_{0}$. The $\Lambda$ parameter of gauge theory is identified as

$$
(R \Lambda)^{4}=\frac{Q_{B}}{16 Q_{F}} .
$$

We can write down the analogues of Nekrasov's formula corresponding to string theory compactified on $\mathbf{F}_{1}$ and $\mathbf{F}_{2}$. These formulas contain some extra framing factors which become trivial in the four-dimensional limit $q \rightarrow 1$.

In all known cases of local Calabi-Yau manifolds, such as local $\mathbf{P}^{2}$ and $\mathbf{F}_{m}$, ChernSimons calculations automatically lead to the form of the Gopakumar-Vafa invariants for the topological string amplitude. In the case of the canonical bundle over Hirzebruch surfaces $\mathbf{F}_{m}$, for instance, its free-energy has an expansion

$$
\mathcal{F}_{\text {top str }}=\sum_{n, m=0}^{\infty} \sum_{g=0}^{\infty} \sum_{k=1}^{\infty} \frac{N_{n, m}^{g}}{k\left(2 \sin k \frac{g_{s}}{2}\right)^{2-2 g}} e^{-k\left(n t_{B}+m t_{F}\right)},
$$

where $g_{s}$ denotes the string coupling constant (related to $q$ as $q=e^{i g_{s}}$ ) and $N_{n, m}^{g}$ are the Gopakumar-Vafa invariants computing the number of BPS states obtained by M2branes wrapped around the two cycles $n B+m F$. Gopakumar-Vafa $(G V)$ invariants have a simple relation to the standard Gromov-Witten(GW) invariants. In examples GV invariants calculated from Chern-Simons theory are in precise agreement with the known GW invariants calculated by using mirror map and holomorphic anomaly, see for instance [21, 22].

What is striking in the method of geometric transition and Nekrasov's formula is that they manage to evaluate the sum over $m$ (winding number around the fiber $F$ ) in (3.42) and the multiple-cover factor $k$ exactly in a very efficient manner. Only the sum over $n$ (winding around the base $B$ ) is left as the sum over the space-time instanton numbers.

We note that the agreement of Nekrasov's formula with the Chern-Simons computation implies that Nekrasov's formula encodes the entire information of topological string 
amplitudes compactified on local $\mathbf{F}_{0}$ including the number of all higher genus curves. By taking suitable limits $R \rightarrow 0$ and/or $\hbar \rightarrow 0$ we recover four dimensional Seiberg-Witten theory or its coupling to graviphoton backgrounds.

\section{Adding matter and blow ups}

According to the prescription of geometric engineering [18, 19, 20, 23] matters in the fundamental representation are obtained by blow ups. By making a blow up at a point on the Hirzebruch surface $\mathbf{F}_{0}$ or $\mathbf{F}_{1}$, we obtain the second del Pezzo surface $d P_{2}^{(0)}$. We also have its "cousin" $d P_{2}^{(1)}$ from blowing up $\mathbf{F}_{2}$ or $\mathbf{F}_{1}$. The reflexive polyhedra for these toric surfaces are No. 5 and 6 , respectively, in the figure 1 of [23]. The dual toric diagrams of these surfaces are one loop pentagon diagrams and topological string amplitudes for $d P_{2}^{(m)}(m=0,1)$ are

$$
\begin{aligned}
Z_{\text {top str }}^{\left(d P_{2}\right)}= & \sum_{R_{1} \cdots R_{5}} W_{R_{5} R_{1}} W_{R_{1} R_{2}} \cdots W_{R_{4} R_{5}} \cdot e^{-\ell_{R_{1}} t_{F}-\ell_{R_{2}} t_{B}-\ell_{R_{3}}\left(t_{F}-t_{E}\right)-\ell_{R_{4}} t_{E}-\ell_{R_{5}}\left(t_{B}+(m+1) t_{F}-t_{E}\right)} \\
& \times(-1)^{\ell_{R_{2}}+\ell_{R_{3}}+\ell_{R_{4}}} \cdot(-1)^{m\left(\ell_{R_{5}}-\ell_{R_{2}}\right)} \cdot q^{-\frac{1}{2}\left(\kappa_{R_{2}}+\kappa_{R_{3}}+\kappa_{R_{4}}\right)} \cdot q^{\frac{m}{2}\left(\kappa_{R_{5}}-\kappa_{R_{2}}\right)}
\end{aligned}
$$
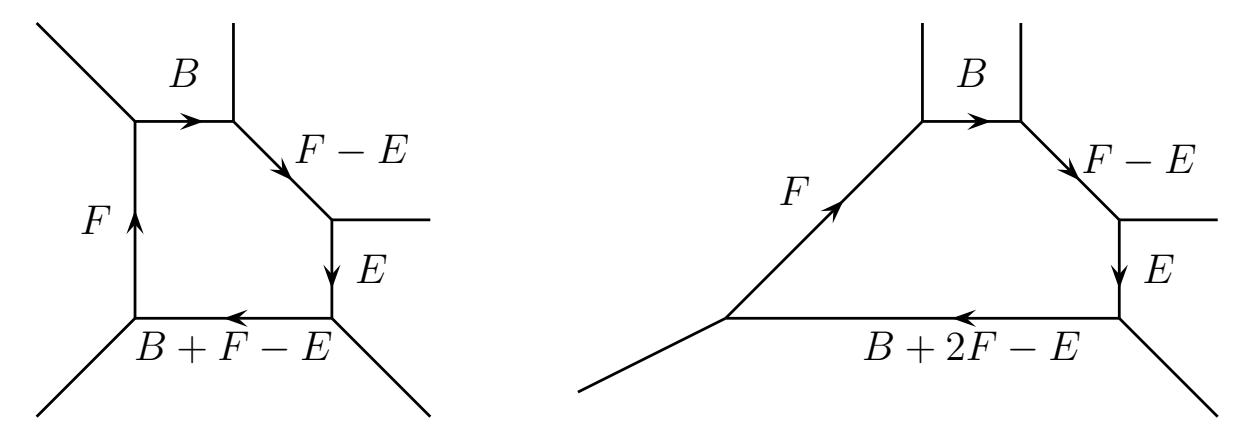

$$
\begin{gathered}
B \cdot B=-(m+1), F \cdot F=0, B \cdot F=+1 \\
E \cdot E=-1, E \cdot F=E \cdot B=0
\end{gathered}
$$

Figure 3 : Toric diagram of the second del Pezzo surface $d P_{2}^{(0)}$ (left) and $d P_{2}^{(1)}$ (right) 
It is convenient to introduce the following building blocks

$$
\begin{aligned}
K_{R_{1} R_{2}}(Q) & :=\sum_{S} Q^{\ell_{S}} W_{R_{1} S}(q) W_{S R_{2}}(q) \\
L_{R_{1} R_{2}}\left(Q_{1}, Q_{2}\right) & :=\sum_{S_{1}, S_{2}} Q_{1}^{\ell_{S_{1}}} Q_{2}^{\ell_{S_{2}}} W_{R_{1} S_{1}}(q) W_{S_{1} S_{2}}(q) W_{S_{2} R_{2}}(q)(-1)^{\ell_{S_{1}}+\ell_{S_{2}}} q^{-\frac{1}{2}\left(\kappa_{S_{1}}+\kappa_{S_{2}}\right)} .
\end{aligned}
$$

Then by cutting the diagram at two internal lines with the Kähler modulus $t_{B}$, we can express the amplitude as

$$
\begin{aligned}
Z_{\text {top str }}^{\left(d P_{2}\right)}=\sum_{R_{1}, R_{2}} & Q_{B}^{\ell_{R_{1}}+\ell_{R_{2}}} Q_{F}^{(m+1) \ell_{R_{2}}} Q_{E}^{-\ell_{R_{2}}}(-1)^{(1-m) \ell_{R_{1}}+m \ell_{R_{2}}} q^{-\frac{1}{2}\left((1-m) \kappa_{R_{1}}+m \kappa_{R_{2}}\right)} \\
& \times K_{R_{1} R_{2}}\left(Q_{F}\right) \cdot L_{R_{1} R_{2}}\left(Q_{F} Q_{E}^{-1}, Q_{E}\right)
\end{aligned}
$$

where $Q_{F}=e^{-t_{F}}, Q_{B}=e^{-t_{B}}$ and $Q_{E}=e^{-t_{E}}$.
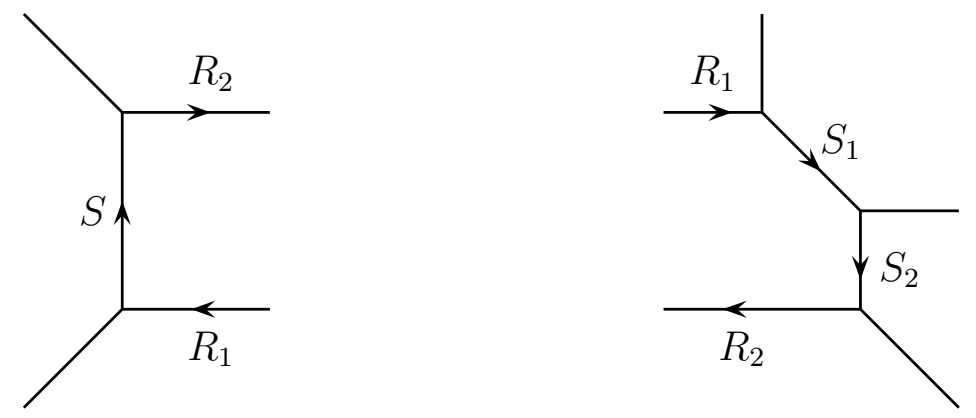

1. The self-intersection of the internal two-cycle of $K_{R_{1} R_{2}}(Q)$ is zero.

2. Two internal two-cycles of $L_{R_{1} R_{2}}\left(Q_{1}, Q_{2}\right)$ are $(-1)$ curves intersecting each other with intersection number +1 .

Figure 4 : Building blocks $K_{R_{1} R_{2}}(Q)$ (left) and $L_{R_{1} R_{2}}\left(Q_{1}, Q_{2}\right)$ (right)

As we have seen in the last section, we have an identity

$$
K_{R_{1} R_{2}}(Q)=W_{R_{1}}(q) W_{R_{2}}(q) \exp \left(\sum_{n=1}^{\infty} \frac{\tilde{f}_{R_{1} R_{2}}\left(q^{n}\right)}{n} Q^{n}\right) .
$$

Let us introduce a similar ansatz for $L_{R_{1} R_{2}}\left(Q_{1}, Q_{2}\right)$;

\section{Proposition 3}

$$
\begin{aligned}
& L_{R_{1} R_{2}}\left(Q_{1}, Q_{2}\right)=W_{R_{1}}(q) W_{R_{2}}(q) \\
& \quad \times \exp \left(\sum_{n=1}^{\infty} \frac{A_{R_{1}}\left(q^{n}\right)}{n} Q_{1}^{n}+\sum_{n=1}^{\infty} \frac{A_{R_{2}}\left(q^{n}\right)}{n} Q_{2}^{n}+\sum_{n=1}^{\infty} \frac{A_{R_{1} R_{2}}\left(q^{n}\right)}{n}\left(Q_{1} Q_{2}\right)^{n}\right) .
\end{aligned}
$$


By comparing coefficients of $Q_{1}, Q_{2}$ and $Q_{1} Q_{2}$ of both sides of the above formula, we find

$$
\begin{aligned}
(-1) W_{R_{1} \square}(q) W_{\square \bullet}(q) W_{\bullet R_{2}}(q) & =W_{R_{1}}(q) W_{R_{2}}(q) A_{R_{1}}(q), \\
(-1) W_{R_{1}} \bullet(q) W_{\bullet \square}(q) W_{\square R_{2}}(q) & =W_{R_{1}}(q) W_{R_{2}}(q) A_{R_{2}}(q), \\
W_{R_{1} \square}(q) W_{\square \square}(q) W_{\square R_{2}}(q) & =W_{R_{1}}(q) W_{R_{2}}(q)\left(A_{R_{1} R_{2}}(q)+A_{R_{1}}(q) A_{R_{2}}(q)\right) .
\end{aligned}
$$

If one uses the relation

$$
W_{R \square}(q)=W_{\square R}(q)=W_{R}(q) W_{\square}^{-1}(q) \widetilde{f}_{R}(q)
$$

one can determine the unknown functions $A_{R_{1}}, A_{R_{2}}, A_{R_{1} R_{2}}$ as

$$
A_{R_{1}}(q)=-\widetilde{f}_{R_{1}}(q), \quad A_{R_{2}}(q)=-\widetilde{f}_{R_{2}}(q)
$$

and

$$
\begin{aligned}
A_{R_{1} R_{2}}(q) & =\widetilde{f}_{R_{1}}(q) \widetilde{f}_{R_{2}}(q)\left(1+W_{\square}^{-2}(q)\right)-\widetilde{f}_{R_{1}}(q) \widetilde{f}_{R_{2}}(q) \\
& =\widetilde{f}_{R_{1} R_{2}}(q)
\end{aligned}
$$

We present a proof of the above formula (4.6) making use of the skew Schur functions 34, 33] in Appendix C.

Recall that the coefficients $C_{k}\left(R_{1}, R_{2}\right)$ were introduced in (3.29) as the expansion coefficients of $f_{R_{1} R_{2}}(q)$. Similarly we define $C_{k}(R)$ as the expansion coefficients of $f_{R}(q)$,

$$
f_{R}(q)=\sum_{k} C_{k}(R) q^{k}
$$

Note that $C_{k}(R)=C_{k}(R, \bullet)$. Using these coefficients, we obtain the following expression for $L_{R_{1} R_{2}}\left(Q_{1}, Q_{2}\right)$;

$$
\begin{gathered}
L_{R_{1} R_{2}}\left(Q_{1}, Q_{2}\right)=W_{R_{1}}(q) W_{R_{2}}(q) \exp \left\{\sum_{n=1}^{\infty} \frac{1}{n} W_{\square}^{2}\left(q^{n}\right)\left(\left(Q_{1} Q_{2}\right)^{n}-Q_{1}^{n}-Q_{2}^{n}\right)\right\} \\
\times \prod_{k} \frac{\left(1-q^{k} Q_{1}\right)^{C_{k}\left(R_{1}\right)}\left(1-q^{k} Q_{2}\right)^{C_{k}\left(R_{2}\right)}}{\left(1-q^{k} Q_{1} Q_{2}\right)^{C_{k}\left(R_{1}, R_{2}\right)}} .
\end{gathered}
$$


Substituting (4.5) and (4.14) into (4.4), one finds

$$
\begin{aligned}
Z_{\text {top str }}^{\left(d P_{2}\right)}= & \exp \left\{\sum_{n=1}^{\infty} \frac{1}{n} W_{\square}^{2}\left(q^{n}\right)\left(2 Q_{F}^{n}-\left(Q_{F} Q_{E}^{-1}\right)^{n}-Q_{E}^{n}\right)\right\} \\
& \times \sum_{R_{1}, R_{2}} Q_{B}^{\ell_{R_{1}}+\ell_{R_{2}}} Q_{F}^{(m+1) \ell_{R_{2}}} Q_{E}^{-\ell_{R_{2}}}(-1)^{(1-m) \ell_{R_{1}}+m \ell_{R_{2}}} q^{-\frac{1}{2}\left((1-m) \kappa_{R_{1}}+m \kappa_{R_{2}}\right)} \\
& \times W_{R_{1}}^{2}(q) W_{R_{2}}^{2}(q) \prod_{k} \frac{\left(1-q^{k}\left(Q_{F} Q_{E}^{-1}\right)\right)^{C_{k}\left(R_{1}\right)}\left(1-q^{k} Q_{E}\right)^{C_{k}\left(R_{2}\right)}}{\left(1-q^{k} Q_{F}\right)^{2 C_{k}\left(R_{1}, R_{2}\right)}}
\end{aligned}
$$

\subsection{Perturbative one loop effective coupling}

In this topological string amplitude, the power of $Q_{B}$ is identified with the space-time instanton number of Yang-Mills field. The first factor of (4.15) is interpreted as the perturbative part of the free energy (the Seiberg-Witten prepotential);

$$
\mathcal{F}_{\text {one loop }}=\sum_{n=1}^{\infty} \frac{1}{n} W_{\square}^{2}\left(q^{n}\right)\left(2 Q_{F}^{n}-\left(Q_{F} Q_{E}^{-1}\right)^{n}-Q_{E}^{n}\right) .
$$

We make the following identification of parameters in the gauge theory side;

$$
Q_{F}=e^{-4 R a}, \quad Q_{E}=e^{-2 R(a-m)} \quad \text { and } \quad Q_{F} Q_{E}^{-1}=e^{-2 R(a+m)},
$$

where $a$ is vacuum expectation value of the scalar in the $\mathcal{N}=2$ vector multiplet and $m$ is the mass of the fundamental matter. $R$ is the radius of the circle. The parameter $\hbar$ is the genus expansion parameter in the topological string and we have

$$
q=e^{-2 \hbar R} .
$$

When we take the limit $q \rightarrow 0$,

$$
W_{\square}^{2}\left(q^{n}\right)=\frac{1}{\left(e^{n \hbar R}-e^{-n \hbar R}\right)^{2}} \rightarrow(2 n \hbar R)^{-2},
$$

and hence

$$
\mathcal{F}_{\text {one loop }} \rightarrow \frac{1}{4 R^{2}} \sum_{n=1}^{\infty} \frac{1}{n^{3}}\left(2 e^{-4 n R a}-e^{-2 n R(a+m)}-e^{-2 n R(a-m)}\right) .
$$


The one loop effective coupling is the second derivative of the prepotential;

$$
\tau_{\text {one loop }}=\frac{\partial^{2} \mathcal{F}_{\text {one loop }}}{\partial a^{2}}=-8 \log \left(1-e^{-4 n R a}\right)+\log \left(1-e^{-2 n R(a+m)}\right)\left(1-e^{-2 n R(a-m)}\right) .
$$

Up to a linear term in $a$ which should be provided from the classical part of the Yukawa couplings we obtain

$$
\tau_{\text {one loop }}=-8 \log \sinh 2 R a+\log \sinh R(a+m)+\log \sinh R(a-m),
$$

which has the correct five-dimensional form of summing up Kaluza-Klein modes. In the decompactified limit $R \rightarrow \infty$ one finds

$$
\tilde{\tau}=\frac{\tau}{R}=-16|a|+|a+m|+|a-m|
$$

This gives the well-known behavior of the coupling constant of uncompactified fivedimensional gauge theory [24].

\subsection{Comparison with Nekrasov's conjecture}

Let us now compare our topological string amplitude with Nekrasov' formula for gauge theory with matter. The instanton part of the amplitude on local del Pezzo surface $d P_{2}$ is

$$
\begin{aligned}
Z_{\text {top str }}^{\left(d P_{2}\right)} & =\sum_{R_{1}, R_{2}} Q_{B}^{\ell_{R_{1}}+\ell_{R_{2}}} Q_{F}^{2 \ell_{R_{2}}} Q_{E}^{-\ell_{R_{2}}}(-1)^{\ell_{R_{2}}} q^{-\frac{1}{2} \kappa_{R_{2}}} W_{R_{1}}^{2}(q) W_{R_{2}}^{2}(q) \\
& \times \prod_{k} \frac{\left(1-q^{k}\left(Q_{F} Q_{E}^{-1}\right)\right)^{C_{k}\left(R_{1}\right)}\left(1-q^{k} Q_{E}\right)^{C_{k}\left(R_{2}\right)}}{\left(1-q^{k} Q_{F}\right)^{2 C_{k}\left(R_{1}, R_{2}\right)}}
\end{aligned}
$$

where we chose $m=1$ for convenience. As in the pure Yang-Mills case, we first replace the summation over $R_{2}$ with that over $R_{2}^{t}$. Then the same computation as before gives

$$
\begin{aligned}
Z_{\text {top str }}^{\left(d P_{2}\right)}= & \sum_{R_{1}, R_{2}}\left(\frac{Q_{B}}{16 Q_{F}}\right)^{\ell_{R_{1}}+\ell_{R_{2}}} Q_{F}^{2 \ell_{R_{2}}} Q_{E}^{-\ell_{R_{2}}}(-1)^{\ell_{R_{2}}} q^{+\frac{1}{2} \kappa_{R_{2}}} \\
& \times \prod_{k}\left(1-q^{k}\left(Q_{F} Q_{E}^{-1}\right)\right)^{+C_{k}\left(R_{1}\right)}\left(1-q^{k} Q_{E}\right)^{+C_{k}\left(R_{2}^{t}\right)} \\
& \times \prod_{\ell, m \in\{1,2\}} \prod_{i, j=1}^{\infty} \frac{\sinh R\left(a_{\ell m}+\hbar\left(\mu_{\ell, i}-\mu_{m, j}+j-i\right)\right)}{\sinh R\left(a_{\ell m}+\hbar(j-i)\right)} .
\end{aligned}
$$


Using the relation $C_{k}\left(R^{t}\right)=C_{-k}(R)$, we have

$$
\begin{aligned}
& \prod_{k}\left(1-q^{k}\left(Q_{F} Q_{E}^{-1}\right)\right)^{+C_{k}\left(R_{1}\right)}\left(1-q^{k} Q_{E}\right)^{+C_{k}\left(R_{2}^{t}\right)} \\
& =2^{\ell_{R_{1}}+\ell_{R_{2}}} q^{\frac{1}{4}\left(\kappa_{R_{1}}-\kappa_{R_{2}}\right)} Q_{F}^{\frac{1}{2} \ell_{R_{1}}} Q_{E}^{\frac{1}{2}\left(\ell_{R_{2}}-\ell_{R_{1}}\right)} \\
& \times \prod_{k}(\sinh R(a+m+\hbar k))^{C_{k}\left(R_{1}\right)}(\sinh R(a-m-\hbar k))^{C_{k}\left(R_{2}\right)} .
\end{aligned}
$$

Hence we obtain

$$
\begin{aligned}
Z_{\text {top str }}^{\left(d P_{2}\right)}= & \sum_{R_{1}, R_{2}}\left(\frac{Q_{B}}{8 Q_{F}}\right)^{\ell_{R_{1}}+\ell_{R_{2}}} Q_{F}^{2 \ell_{R_{2}}+\frac{1}{2} \ell_{R_{1}}} Q_{E}^{-\frac{1}{2}\left(\ell_{R_{1}}+\ell_{R_{2}}\right)} q^{\frac{1}{4}\left(\kappa_{R_{1}}+\kappa_{R_{2}}\right)} \\
& \times \prod_{k}(\sinh R(a+m+\hbar k))^{C_{k}\left(R_{1}\right)}(\sinh R(-a+m+\hbar k))^{C_{k}\left(R_{2}\right)} \\
& \times \prod_{\ell, m \in\{1,2\}} \prod_{i, j=1}^{\infty} \frac{\sinh R\left(a_{\ell m}+\hbar\left(\mu_{\ell, i}-\mu_{m, j}+j-i\right)\right)}{\sinh R\left(a_{\ell m}+\hbar(j-i)\right)}
\end{aligned}
$$

In the four dimensional limit $R \rightarrow 0$ last two factors give

$$
\begin{aligned}
& \frac{1}{R^{3\left(\ell_{R_{1}}+\ell_{R_{2}}\right)}} \prod_{k}(a+m+\hbar k)^{C_{k}\left(R_{1}\right)}(-a+m+\hbar k)^{C_{k}\left(R_{2}\right)} \\
& \times \prod_{\ell, m \in\{1,2\}} \prod_{i, j=1}^{\infty} \frac{a_{\ell m}+\hbar\left(\mu_{\ell, i}-\mu_{m, j}+j-i\right)}{a_{\ell m}+\hbar(j-i)}
\end{aligned}
$$

We note that $Q_{B}=2(R \Lambda)^{3}$ and the powers of $R$ cancel each other.

On the other hand Nekrasov's formula for $S U(2)$ theory with $N_{f}=1$ is given by (in our notation);

$$
\begin{aligned}
& (\hbar \Lambda)^{\ell_{R_{1}}+\ell_{R_{2}}} \prod_{(\ell, i)} \frac{\Gamma\left(\frac{a_{\ell}+m}{\hbar}+1+\mu_{\ell, i}-i\right)}{\Gamma\left(\frac{a_{\ell}+m}{\hbar}+1-i\right)} \\
& \quad \times \prod_{\ell, m \in\{1,2\}} \prod_{i, j=1}^{\infty} \frac{a_{\ell m}+\hbar\left(\mu_{\ell, i}-\mu_{m, j}+j-i\right)}{a_{\ell m}+\hbar(j-i)} .
\end{aligned}
$$

Since we have seen that the pure gauge part agrees in section 3, we will examine the additional factor of matter contribution (first line of (4.29));

$$
\prod_{i=1}^{d\left(\mu^{R_{1}}\right)} \prod_{j=1}^{\mu_{1, i}}(a+m+\hbar(j-i)) \times \prod_{i=1}^{d\left(\mu^{R_{2}}\right)} \prod_{j=1}^{\mu_{2, i}}(-a+m+\hbar(j-i))
$$


where we have used $\Gamma(z+1)=z \Gamma(z)$ and absorbed the powers of $\hbar$. If we recall that the generation function of $C_{k}(R)$ is

$$
f_{R}(q)=\sum_{i=1}^{d\left(\mu^{R}\right)} \sum_{j=1}^{\mu_{i}} q^{j-i}
$$

we see that the four dimensional limit of our amplitude in fact exactly agrees with Nekrasov's formula.

In this comparison we have considered the amplitude for $d P_{2}^{(1)}$. In the four dimensional limit the amplitudes for $d P_{2}^{(0)}$ and $d P_{2}^{(1)}$ are simply related by the sign flip of the mass parameter $m \rightarrow-m$.

\subsection{Four dimensional limit and Seiberg-Witten prepotential}

Let us next check if our amplitude reproduces the Seiberg-Witten prepotential of $S U(2)$ theory with a single hypermultiplet.

We first recall the definition of instanton expansion of the free-energy

$$
\begin{aligned}
& \log Z_{\text {top str }}\left(Q_{B}, Q_{F}, Q_{E}, q\right)=\mathcal{F}_{\text {one loop }}\left(Q_{F}, Q_{E}, q\right)+\mathcal{F}_{\text {inst }}\left(Q_{B}, Q_{F}, Q_{E}, q\right) \\
& \mathcal{F}_{\text {inst }}\left(Q_{B}, Q_{F}, Q_{E}, q\right)=\sum_{k=1}^{\infty} \sum_{n=1}^{\infty} \frac{1}{n} Q_{B}^{n k} \mathcal{F}_{k}\left(Q_{F}^{n}, Q_{E}^{n}, q^{n}\right) \\
& \mathcal{F}_{k}\left(Q_{F}, Q_{E}, q\right)=\sum_{g=0}^{\infty} \frac{1}{\left(2 \sin \frac{g_{s}}{2}\right)^{2-2 g}} f_{g}^{(k)}\left(Q_{F}, Q_{E}\right)
\end{aligned}
$$

where $f_{g}^{(k)}$ gives the genus- $g k$-instanton amplitudes and the string coupling constant $g_{s}$ is related to $q$ as

$$
q=e^{i g_{s}}=e^{-2 \hbar R}
$$

Note that (4.34) defines the genus expansion of the Gopakumar-Vafa type. When we take the four-dimensional limit, it is converted into an ordinary genus expansion in powers of $\hbar$. 
By explicit computations of (4.15) we find the following results for genus zero instanton amplitudes

$$
\begin{aligned}
& f_{g=0}^{(1)}\left(Q_{F}, Q_{E}\right)=\frac{N_{0}^{(1)}}{\left(Q_{F}-1\right)^{2}}, \\
& f_{g=0}^{(2)}\left(Q_{F}, Q_{E}\right)=\frac{N_{0}^{(2)}}{\left(Q_{F}+1\right)^{2}\left(Q_{F}-1\right)^{6}}, \\
& f_{g=0}^{(3)}\left(Q_{F}, Q_{E}\right)=\frac{N_{0}^{(3)}}{\left(Q_{F}^{2}+Q_{F}+1\right)^{2}\left(Q_{F}-1\right)^{10}},
\end{aligned}
$$

where the numerators are

$$
\begin{aligned}
N_{0}^{(1)}=- & \left(Q_{F}+1\right)+2 Q_{F} Q_{E}^{-1} \\
N_{0}^{(2)}=( & \left.6 Q_{F}^{2}+8 Q_{F}+6\right) Q_{F}^{2}-\left(5 Q_{F}^{3}+15 Q_{F}^{2}+15 Q_{F}+5\right) Q_{F}^{2} Q_{E}^{-1} \\
& +\left(6 Q_{F}^{2}+8 Q_{F}+6\right) Q_{F}^{3} Q_{E}^{-2} \\
N_{0}^{(3)}=- & \left(27 Q_{F}^{5}+70 Q_{F}^{4}+119 Q_{F}^{3}+119 Q_{F}^{2}+70 Q_{F}+27\right) Q_{F}^{3} \\
& +\left(32 Q_{F}^{6}+144 Q_{F}^{5}+288 Q_{F}^{4}+368 Q_{F}^{3}+288 Q_{F}^{2}+144 Q_{F}+32\right) Q_{F}^{3} Q_{E}^{-1} \\
& -\left(7 Q_{F}^{7}+79 Q_{F}^{6}+216 Q_{F}^{5}+346 Q_{F}^{4}+346 Q_{F}^{3}+216 Q_{F}^{2}+79 Q_{F}+7\right) Q_{F}^{3} Q_{E}^{-2} \\
& +\left(8 Q_{F}^{7}+46 Q_{F}^{6}+100 Q_{F}^{5}+124 Q_{F}^{4}+100 Q_{F}^{3}+46 Q_{F}^{2}+8 Q_{F}\right) Q_{F}^{3} Q_{E}^{-3}
\end{aligned}
$$

The coefficients of the Taylor expansion of $f_{g=0}^{(k)}$ give the integer invariants of GopakumarVafa. We find a perfect agreement with Table 3 in [23. (Their Kähler moduli $t_{i}$ are related to ours by $t_{1}=t_{F}, t_{2}=t_{B}, t_{3}=t_{E}+t_{F}$.) We note that when the mass $m$ goes to infinity, $Q_{E} \rightarrow 0$ and the most singular part in this limit reproduces the result of the Hirzebruch surface (we redefine $Q_{B} Q_{F} / Q_{E}$ as $Q_{B}$ for the Hirzebruch surface).

Substituting (4.17), we find

$$
\begin{aligned}
f_{g=0}^{(1)}\left(Q_{F}, Q_{E}\right) & \approx \frac{1}{4} \frac{m}{a^{2}} R^{-1}, \quad f_{g=0}^{(2)}\left(Q_{F}, Q_{E}\right) \approx \frac{1}{2^{10}} \frac{5 m^{2}-3 a^{2}}{a^{6}} R^{-4} \\
f_{g=0}^{(3)}\left(Q_{F}, Q_{E}\right) & \approx \frac{1}{3 \cdot 2^{13}} \frac{m\left(9 m^{2}-7 a^{2}\right)}{a^{10}} R^{-7} .
\end{aligned}
$$

At genus zero we have a contribution $(2 \hbar R)^{-2}$ from $\sin ^{-2}(q / 2)$ and we identify

$$
Q_{B}=2(R \Lambda)^{3}
$$


then in the four-dimensional limit $R \rightarrow 0$ we have

$$
\begin{aligned}
& f_{g=0}^{(1)}\left(Q_{F}, Q_{E}\right) \cdot Q_{B} \rightarrow \frac{1}{2^{3}} \frac{m}{a^{2}} \Lambda^{3}, \quad f_{g=0}^{(2)}\left(Q_{F}, Q_{E}\right) \cdot Q_{B}^{2} \rightarrow \frac{1}{2^{10}} \frac{5 m^{2}-3 a^{2}}{a^{6}} \Lambda^{6}, \\
& f_{g=0}^{(3)}\left(Q_{F}, Q_{E}\right) \cdot Q_{B}^{3} \rightarrow \frac{1}{3 \cdot 2^{12}} \frac{m\left(9 m^{2}-7 a^{2}\right)}{a^{10}} \Lambda^{9} .
\end{aligned}
$$

The first two expressions agree with Eq.(5.5) of [25]. After a rescaling

$$
m \rightarrow \frac{m}{\sqrt{2}}, \quad \Lambda \rightarrow \frac{\Lambda}{\sqrt{2}}
$$

we obtain

$$
\mathcal{F}_{1}=\frac{1}{2^{5}} \frac{m}{a^{2}} \Lambda^{3}, \quad \mathcal{F}_{2}=\left(\frac{5}{2^{14}} \frac{m^{2}}{a^{6}}-\frac{3}{2^{13}} \frac{1}{a^{4}}\right) \Lambda^{6}, \quad \mathcal{F}_{3}=\left(\frac{3}{2^{18}} \frac{m^{3}}{a^{10}}-\frac{7}{3 \cdot 2^{17}} \frac{1}{a^{8}}\right) \Lambda^{9} .
$$

Then we find an agreement with [26] (Addendix D) (up to an overall normalization factor of 2). The decoupling limit $m \Lambda^{3} \equiv \Lambda_{0}^{4}$ and $m \rightarrow \infty$ gives the instanton expansion of the prepotential of pure $S U(2)$ gauge theory.

\subsection{Genus one amplitude and coupling to gravity}

In type II string theory topological string amplitudes at higher genus compute the (Ftype) couplings of four-dimensional gauge theory with gravitational background field [27. In particular the genus one amplitude $\mathcal{F}^{(1)}$ contributes to the following coupling;

$$
\frac{1}{2} \mathcal{F}^{(1)}(a, m)\left(\chi-\frac{3}{2} \sigma\right),
$$

where $\chi$ and $\sigma$ are the Euler characteristic and the signature of the four manifold.

Again by explicit calculations of (4.15) we can derive that the $k$-instanton parts of

the free energy $f_{g=1}^{(k)}\left(Q_{F}, Q_{E}\right)$ at genus one. They are given by

$$
\begin{aligned}
& f_{g=1}^{(1)}\left(Q_{F}, Q_{E}\right)=0, \\
& f_{g=1}^{(2)}\left(Q_{F}, Q_{E}\right)=\frac{N_{1}^{(2)}}{\left(Q_{F}+1\right)^{2}\left(Q_{F}-1\right)^{8}}, \\
& f_{g=1}^{(3)}\left(Q_{F}, Q_{E}\right)=\frac{N_{1}^{(3)}}{\left(Q_{F}^{2}+Q_{F}+1\right)^{2}\left(Q_{F}-1\right)^{12}},
\end{aligned}
$$


where the numerators are

$$
\begin{aligned}
N_{1}^{(2)}= & \left(9 Q_{F}^{2}+14 Q_{F}+9\right) Q_{F}^{3}-\left(8 Q_{F}^{3}+24 Q_{F}^{2}+24 Q_{F}+8\right) Q_{F}^{3} Q_{E}^{-1} \\
& +\left(9 Q_{F}^{2}+14 Q_{F}+9\right) Q_{F}^{4} Q_{E}^{-2} \\
N_{1}^{(3)}= & -\left(10 Q_{F}^{7}+188 Q_{F}^{6}+508 Q_{F}^{5}+830 Q_{F}^{4}+830 Q_{F}^{3}+508 Q_{F}^{2}+188 Q_{F}+10\right) Q_{F}^{3} \\
+ & \left(9 Q_{F}^{8}+254 Q_{F}^{7}+1033 Q_{F}^{6}+2030 Q_{F}^{5}+2564 Q_{F}^{4}+2030 Q_{F}^{3}+1033 Q_{F}^{2}+254 Q_{F}+9\right) Q_{F}^{3} Q_{E}^{-1} \\
- & \left(72 Q_{F}^{8}+584 Q_{F}^{7}+1536 Q_{F}^{6}+2416 Q_{F}^{5}+2416 Q_{F}^{4}+1536 Q_{F}^{3}+584 Q_{F}^{2}+72 Q_{F}\right) Q_{F}^{3} Q_{E}^{-2} \\
+ & \left(68 Q_{F}^{8}+336 Q_{F}^{7}+692 Q_{F}^{4}+880 Q_{F}^{5}+692 Q_{F}^{4}+336 Q_{F}^{3}+68 Q_{F}^{2}\right) Q_{F}^{3} Q_{E}^{-3} .
\end{aligned}
$$

The coefficients of the Taylor expansion of $f_{g=1}^{(k)}\left(Q_{F}, Q_{E}\right)$ give the genus-one integer invariants of Gopakumar-Vafa. We note that there is no contribution at genus one from one-instanton sector. In the decoupling limit; $Q_{E} \rightarrow 0$, the most singular term reproduces the genus one amplitudes of the Hirzebruch surface. To take the four dimensional limit, we substitute $Q_{F}=\exp (-4 R a), Q_{E}=\exp (-2 R(a-m))$ and $Q_{B}=2(R \Lambda)^{3}$ as in the genus zero computation. Then we have

$$
\begin{aligned}
& f_{g=1}^{(2)}\left(Q_{F}, Q_{E}\right) \cdot Q_{B}^{2} \rightarrow \frac{1}{2^{11}} \frac{4 m^{2}-3 a^{2}}{a^{8}} \Lambda^{6}, \\
& f_{g=1}^{(3)}\left(Q_{F}, Q_{E}\right) \cdot Q_{B}^{3} \rightarrow \frac{1}{3 \cdot 2^{13}} \frac{m\left(32 m^{2}-27 a^{2}\right)}{a^{12}} \Lambda^{9} .
\end{aligned}
$$

We can compare the above result with the gravitational coupling in the twisted $\mathcal{N}=2$ $S U(2)$ Yang-Mills theory with a fundamental matter. For pure Yang-Mills theory such comparison has been made in [28, 29]. The anomaly cancellation of the measure of the $u$ plane integral of the Seiberg-Witten theory predicts the following coupling to the gravity 30;

$$
\frac{\chi}{2} \log \left(\frac{1}{\Lambda} \frac{d u}{d a}\right)+\frac{\sigma}{8} \log \left(\frac{\Delta}{\Lambda^{6}}\right)
$$

Here $u$ is the coordinate on the moduli space of the Coulomb branch, $a(u)$ is the period of the Seiberg-Witten differential on the $\alpha$ cycle and $\Delta$ is the discriminant of the SeibergWitten curve. As we know, the curve for $N_{f}=1$ is given by

$$
y^{2}=\left(x^{2}-u\right)^{2}-\Lambda^{3}(x+m)
$$


with the discriminant

$$
\Delta=27 \Lambda^{6}+256 \Lambda^{3} m^{3}-288 \Lambda^{3} m u-256 m^{2} u^{2}+256 u^{3} .
$$

The inversion of the period $a=a(u)$ in the weak coupling region is [26]

$$
u(a)=2 a^{2}+\frac{m}{16 a^{2}} \Lambda^{3}-\frac{3}{2048 a^{4}} \Lambda^{6}+\frac{5 m^{2}}{4096 a^{6}} \Lambda^{6}-\frac{7 m}{65536 a^{8}} \Lambda^{9}+\frac{9 m^{3}}{131072 a^{10}} \Lambda^{9}+\cdots .
$$

The twisted theory and the physical theory agree on the hyperKähler manifold and we will take $K 3$ surface with $\chi=24$ and $\sigma=-16$. Then comparing the gravitational coupling of both theories, we find

$$
\mathcal{F}^{(1)}=\frac{1}{2} \log \left(\frac{1}{\Lambda} \frac{d u}{d a}\right)-\frac{1}{12} \log \left(\frac{\Delta}{\Lambda^{6}}\right) .
$$

Substituting the above data obtained from the Seiberg-Witten curve we compute

$$
\mathcal{F}^{(1)}=\frac{1}{12} \log \frac{4 a^{2}}{2 a^{2}-m^{2}}+\frac{1}{2^{14}} \frac{2 m^{3}-3 a^{2}}{a^{8}} \Lambda^{6}+\frac{1}{3 \cdot 2^{18}} \frac{m\left(16 m^{2}-27 a^{2}\right)}{a^{12}} \Lambda^{9}+\cdots
$$

After the same rescaling of $m$ and $\Lambda$ we have used in the genus zero, we find a precise agreement with our prediction (4.53), (4.54).

\section{Discussions}

In this paper we have used geometric transition and computed all genus topological string amplitude on the local Calabi-Yau manifold $K_{\mathbf{F}_{0}}$ and have shown that the results agree with the Nekrasov's formula for $\mathcal{N}=2$ gauge theory with parameters $\beta$, $\hbar$ being kept non-zero. When one takes the four-dimensional limit $\beta=R \rightarrow 0$, one recovers the wellknown Seiberg-Witten theory and also its coupling to external graviphoton background.

We have also computed the amplitude for the local 2nd del Pezzo surface and have shown that the result agrees with Nekrasov's formula for gauge theory with a matter hypermultiplet.

We have provided proofs for formulas involving Hopf-link invariants which appeared in Chern-Simons calculations. In this sense our derivations are rigorous given the starting 
point of Chern-Simons amplitudes. We feel, however, that there is at present a lack of efficient mathematical techniques in handling formulas containing Chern-Simons invariants and a need for developing more powerful machinery. One obvious candidate is the operator method of two-dimensional CFT which is now being developed in [17, 33].

In a future publication we would like to study more details of topological string theory by making use of operator techniques.

\section{Acknowledgements}

H.K. would like to thank M. Mulase, H. Ohta and A. Tsuchiya for helpful discussions. Our research is supported in part by the Grant-in-Aid for Scientific Research (No.15540253 and No. 14570073) from Japan ministry of education, culture and sports.

\section{Notes added}

After this paper was submitted to e-print archives, a new paper has appeared [35] which is closely related to ours. The formula (2.4) has appeared in the mathematics literature [36], where the degree of the normal bundle is used instead of the self-intersection number. We would like to thank the referee for this comment.

\section{Appendix A : Chern-Simons invariants}

The primary fields of $U(N)$ WZW theory are associated with highest weight representation at level $N+k$. The space of conformal blocks of WZW theory on torus is the set of such states $|R\rangle$. The generators $T$ and $S$ of $S L(2, \mathbf{Z})$ act on the space of conformal blocks. From the relation of the space of conformal blocks and the Hilbert space of the Chern-Simons theory on a 3-manifold whose boundary is a torus the matrix element

$W_{R_{1} R_{2}}=\left\langle\bar{R}_{1}\left|S^{-1}\right| R_{2}\right\rangle$ is the Chern-Simons invariant of the Hopf link in $S^{3}$ with linking 
number +1 [31]. It is expressed in terms of the $q$-numbers

$$
[x]=q^{\frac{x}{2}}-q^{-\frac{x}{2}},
$$

where $q=\exp \left(\frac{2 \pi i}{N+k}\right)$. Let $\mu_{j}$ be the number of boxes in the $j$-th row of the Young diagram $\mu^{R}$ associated with the representation $R$. We define two integers $\ell_{R}$ and $\kappa_{R}$ by

$$
\ell_{R}=\sum_{j=1}^{d\left(\mu^{R}\right)} \mu_{j}, \quad \kappa_{R}=\ell_{R}+\sum_{j=1}^{d\left(\mu^{R}\right)} \mu_{j}\left(\mu_{j}-2 j\right)
$$

where $d\left(\mu^{R}\right)$ is the number of rows of $\mu^{R}$.

Let $\hat{\mu}^{R}$ denote the transposed Young diagram of $\mu^{R}$ obtained by exchanging rows and columns. According to the Jacobi-Trudy formula the Schur polynomial $s_{\mu^{R}}$ in the variables $\left(x_{1}, \cdots, x_{N}\right)$ corresponding to the Young diagram $\mu^{R}$ can be expressed in terms of the elementary symmetric polynomials $e_{i}\left(x_{1}, \cdots, x_{N}\right)$ as follows;

$$
s_{\mu^{R}}=\operatorname{det}_{r \times r} M_{\mu^{R}},
$$

where $r=d\left(\hat{\mu}^{R}\right)$ and $M_{i j}=e_{\hat{\mu}_{i}+j-i}$. This formula is extended to the generalized Schur polynomial $s_{\mu^{R}}(E(t))$ for any formal power series $E(t)=1+\sum_{n=1}^{\infty} a_{n} t^{n}$ by replacing the generating function of the elementary symmetric polynomials $P(t)=\prod_{i=1}^{N}\left(1+x_{i} t\right)=$ $1+\sum_{n=1}^{\infty} e_{n} t^{n}$ by $E(t)$. Then the matrix element $W_{R_{1}, R_{2}}$ is given by [32]

$$
W_{R_{1}, R_{2}}(q)=\left(\operatorname{dim}_{q} R_{1}\right) \cdot q^{\ell_{R_{2}} / 2} \cdot s_{\mu^{R_{2}}}\left(E_{\mu^{R_{1}}}(t)\right)
$$

where

$$
\operatorname{dim}_{q} R=\prod_{1 \leq i<j \leq d\left(\mu^{R}\right)} \frac{\left[\mu_{i}-\mu_{j}+j-i\right]}{[j-i]} \prod_{i=1}^{d\left(\mu^{R}\right)} \prod_{k=1}^{\mu_{i}} \frac{1}{\left[k-i+d\left(\mu^{R}\right)\right]},
$$

is called quantum dimension of $R$ and equal to the Chern-Simons invariant of the unknot carrying representation $R$. The polynomial $E_{\mu^{R}}$ is defined by

$$
E_{\mu^{R}}(t)=\left(\prod_{j=1}^{d\left(\mu^{R}\right)} \frac{1+q^{\mu_{j}-j} t}{1+q^{-j} t}\right) E_{\emptyset}(t), \quad E_{\emptyset}(t)=1+\sum_{n=1}^{\infty}\left(\prod_{i=1}^{n} \frac{1}{q^{i}-1}\right) t^{n} .
$$


When $R_{1}$ is the trivial representation, (A.4) implies that the quantum dimension is also expressed by the Schur functions;

$$
W_{\bullet R}=W_{R}=\operatorname{dim}_{q} R=q^{\ell_{R} / 2} s_{\mu^{R}}\left(E_{\emptyset}(t)\right) .
$$

\section{Appendix B : Proof of Proposition 2}

We first recall the definition of $C_{k}\left(R_{1}, R_{2}\right)$;

$$
\sum_{k} C_{k}\left(R_{1}, R_{2}\right) q^{k}=\left(q+q^{-1}-2\right) f_{R_{1}}(q) f_{R_{2}}(q)+f_{R_{1}}(q)+f_{R_{2}}(q),
$$

where $f_{R}(q)$ is given by (4.31). To simplify the notation let us use the following abbreviation;

$$
d_{1}=d\left(\mu^{R_{1}}\right), \quad d_{2}=d\left(\mu^{R_{2}}\right), \quad \mu_{1, i}=\mu_{i}, \quad \mu_{2, j}=\nu_{j},
$$

and set $R=1$ for simplicity. Paying attention to the fact that the second argument is the transpose of $R_{2}$,

$$
\begin{aligned}
\sum_{k} C_{k}\left(R_{1}, R_{2}^{t}\right) q^{k} & =f_{R_{1}}(q) \sum_{j=1}^{d_{2}} \sum_{i=1}^{\nu_{j}}\left(q^{1+j-i}+q^{-1+j-i}-2 q^{j-i}\right)+f_{R_{1}}(q)+\left(\sum_{j=1}^{d_{2}} \sum_{i=1}^{\nu_{j}} q^{j-i}\right) \\
& =f_{R_{1}}(q) q^{d_{2}}+f_{R_{1}}(q) \sum_{j=1}^{d_{2}}\left(q^{j-\nu_{j}-1}-q^{j-\nu_{j}}\right)+\left(\sum_{j=1}^{d_{2}} \sum_{i=1}^{\nu_{j}} q^{j-i}\right) \cdot \quad \text { (B.3) }
\end{aligned}
$$

Hence we obtain

$$
\begin{aligned}
& \prod_{k} \frac{1}{\sinh (2 a+\hbar k)^{C_{k}\left(R_{1}, R_{2}^{t}\right)}} \\
= & \prod_{i=1}^{d_{1}} \prod_{j=1}^{\mu_{i}} \frac{1}{\sinh \left(2 a+\hbar\left(j-i+d_{2}\right)\right)} \times \prod_{i=1}^{d_{1}} \prod_{j=1}^{d_{2}} \prod_{k=1}^{\mu_{i}} \frac{\sinh \left(2 a+\hbar\left(k-i+j-\nu_{j}\right)\right)}{\sinh \left(2 a+\hbar\left(k-i+j-1-\nu_{j}\right)\right)} \\
& \times \prod_{j=1}^{d_{2}} \prod_{i=1}^{\nu_{j}} \frac{1}{\sinh (2 a+\hbar(j-i))} \\
= & \prod_{i=1}^{d_{1}} \prod_{j=1}^{\mu_{i}} \frac{1}{\sinh \left(2 a+\hbar\left(j-i+d_{2}\right)\right)} \times \prod_{i=1}^{d_{1}} \prod_{j=1}^{d_{2}} \frac{\sinh \left(2 a+\hbar\left(\mu_{i}-\nu_{j}+j-i\right)\right)}{\sinh \left(2 a+\hbar\left(j-i-\nu_{j}\right)\right)} \\
& \times \prod_{j=1}^{d_{2}} \prod_{i=1}^{\nu_{j}} \frac{1}{\sinh (2 a+\hbar(j-i))} .
\end{aligned}
$$


On the other hand, splitting the set of indices $(i, j) \in \mathbf{Z}_{+} \times \mathbf{Z}_{+}$into four disjoint sets; $\left\{1 \leq i \leq d_{1}, 1 \leq j \leq d_{2}\right\} \cup\left\{d_{1}<i<\infty, 1 \leq j \leq d_{2}\right\} \cup\left\{1 \leq i \leq d_{1}, d_{2}<j<\infty\right\} \cup\left\{d_{1}<\right.$ $\left.i<\infty, d_{2}<j<\infty\right\}$, we can compute the expression in the gauge theory side;

$$
\begin{aligned}
& \prod_{i, j=1}^{\infty} \frac{\sinh \left(2 a+\hbar\left(\mu_{i}-\nu_{j}+j-i\right)\right)}{\sinh (2 a+\hbar(j-i))} \\
= & \prod_{i=1}^{d_{1}} \prod_{j=1}^{d_{2}} \frac{\sinh \left(2 a+\hbar\left(\mu_{i}-\nu_{j}+j-i\right)\right)}{\sinh (2 a+\hbar(j-i))} \times \prod_{j=1}^{d_{2}} \prod_{i=1}^{\nu_{j}} \frac{1}{\sinh \left(2 a+\hbar\left(j-i-d_{1}\right)\right)} \\
& \times \prod_{i=1}^{d_{1}} \prod_{j=1}^{\mu_{i}} \frac{1}{\sinh \left(2 a+\hbar\left(j-i+d_{2}\right)\right)}
\end{aligned}
$$

To compare with (B.4) we can further transform the first two factors into

$$
\begin{aligned}
& \prod_{i=1}^{d_{1}} \prod_{j=1}^{d_{2}} \sinh \left(2 a+\hbar\left(\mu_{i}-\nu_{j}+j-i\right)\right) \times \prod_{j=1}^{d_{2}} \prod_{i=1}^{d_{1}+\nu_{j}} \frac{1}{\sinh (2 a+\hbar(j-i))} \\
= & \prod_{i=1}^{d_{1}} \prod_{j=1}^{d_{2}} \frac{\sinh \left(2 a+\hbar\left(\mu_{i}-\nu_{j}+j-i\right)\right)}{\sinh \left(2 a+\hbar\left(j-i-\nu_{j}\right)\right)} \times \prod_{j=1}^{d_{2}} \prod_{i=1}^{\nu_{j}} \frac{1}{\sinh (2 a+\hbar(j-i))} .
\end{aligned}
$$

Thus we find that the final expression is exactly the same as that of topological string side (B.4)!

\section{Appendix C : Proof of Proposition 3}

We recall that the Hopf link invariants are given by

$$
W_{R_{1}, R_{2}}(q)=W_{R_{1}}(q) q^{\frac{\ell_{R_{2}}}{2}} s_{\mu^{R_{2}}}\left(E_{\mu^{R_{1}}}\right)=W_{R_{1}}(q) s_{R_{2}}\left(q^{\mu^{R_{1}}+\rho}\right)
$$

where $q^{\mu+\rho}$ means that we make the following substitution;

$$
s_{R}\left(x_{i}=q^{\mu_{i}-i+\frac{1}{2}}\right)
$$

Here we have made a shift of $1 / 2$ of the power of $q$ from (3.22) in order to absorb the factor $q^{\ell_{R_{2}} / 2}$. By taking $R_{1}=\bullet$, we obtain

$$
W_{R}(q)=s_{R}\left(q^{\rho}\right)=q^{\kappa_{R} / 2} s_{R^{t}}\left(q^{\rho}\right)
$$


and hence

$$
W_{R_{1} R_{2}}(q)=s_{R_{1}}\left(q^{\rho}\right) s_{R_{2}}\left(q^{\mu^{R_{1}}+\rho}\right)
$$

According to [17] the topological vertex is given by

$$
C_{R_{1}, R_{2}, R_{3}}=q^{\kappa_{R_{2}} / 2+\kappa_{R_{3}} / 2} \sum_{Q_{1}, Q_{2}} N_{Q_{1} Q_{2}}^{R_{1} R_{3}^{t}} \frac{W_{R_{2}^{t} Q_{1}} W_{R_{2} Q_{2}}}{W_{R_{2}}}
$$

where

$$
N_{Q_{1} Q_{2}}^{R_{1} R_{3}^{t}}=\sum_{Q} N_{Q Q_{1}}^{R_{1}} N_{Q Q_{2}}^{R_{3}^{t}} .
$$

Recall the definition of the skew Schur function 34

$$
s_{R_{1} / R}(x)=\sum_{R_{2}} N_{R R_{2}}^{R_{1}} s_{R_{2}}(x)
$$

Hence we have

$$
\begin{aligned}
C_{R_{1}, R_{2}, R_{3}} & =q^{\kappa_{R_{2}} / 2+\kappa_{R_{3}} / 2} \sum_{Q_{1}, Q_{2}, Q_{3}} N_{Q_{3} Q_{1}}^{R_{1}} N_{Q_{3} Q_{2}}^{R_{3}^{t}} s_{R_{2}^{t}}\left(q^{\rho}\right) s_{Q_{1}}\left(q^{\mu^{R_{2}^{t}}+\rho}\right) s_{Q_{2}}\left(q^{\left.\mu^{R_{2}}+\rho\right)}\right. \\
& =q^{\kappa_{R_{2}} / 2+\kappa_{R_{3}} / 2} \sum_{Q_{3}} s_{R_{2}^{t}}\left(q^{\rho}\right) s_{R_{1} / Q_{3}}\left(q^{\mu^{R_{2}^{t}}+\rho}\right) s_{R_{3}^{t} / Q_{3}}\left(q^{\mu^{R_{2}}+\rho}\right)
\end{aligned}
$$

This is slightly different from the expression given in 33 , but this form is more convenient in the following. By taking $R_{2}=\bullet$ and using the cyclic symmetry of $C_{R_{1}, R_{2}, R_{3}}$, we obtain

$$
C_{\bullet, R_{3}, R_{1}}=q^{\kappa_{R_{3}} / 2} \sum_{Q} s_{R_{1} / Q}\left(q^{\rho}\right) s_{R_{3}^{t} / Q}\left(q^{\rho}\right)
$$

Hence,

$$
W_{R_{1} R_{2}}=q^{\kappa_{R_{2}} / 2} C_{\bullet R_{1} R_{2}^{t}}=q^{\kappa_{R_{1}} / 2+\kappa_{R_{2}} / 2} \sum_{Q} s_{R_{2}^{t} / Q}\left(q^{\rho}\right) s_{R_{1}^{t} / Q}\left(q^{\rho}\right) .
$$

Thus we have obtained a manifestly symmetric form of $W_{R_{1} R_{2}}$. On the other hand, if we take $R_{3}=\bullet$, only the trivial representation contributes for the summation over $Q$ and

$$
C_{R_{1}, R_{2}, \bullet}=s_{R_{2}}\left(q^{\rho}\right) s_{R_{1}}\left(q^{\mu^{R_{2}^{t}}+\rho}\right)
$$

Hence,

$$
W_{R_{1} R_{2}}=q^{\kappa_{R_{2}} / 2} s_{R_{2}^{t}}\left(q^{\rho}\right) s_{R_{1}}\left(q^{\mu^{R_{2}}+\rho}\right)=s_{R_{2}}\left(q^{\rho}\right) s_{R_{1}}\left(q^{\mu^{R_{2}}+\rho}\right)
$$


After the exchange of $R_{1}$ and $R_{2}$ we recover our original expression of $W_{R_{1} R_{2}}$.

When the topological vertices are expressed in terms of the (skew) Schur functions, a summation over representations may be performed by using the following formulas [34];

$$
\begin{aligned}
& \sum_{R} s_{R^{t}}(x) s_{R}(y)=\prod_{i, j \geq 1}\left(1+x_{i} y_{j}\right) \\
& \sum_{R} s_{R}(x) s_{R}(y)=\prod_{i, j \geq 1}\left(1-x_{i} y_{j}\right)^{-1}
\end{aligned}
$$

Next we introduce the "relative" hook length by

$$
h_{R_{1} R_{2}}(i, j):=\mu_{i}^{R_{1}}-i+\mu_{j}^{R_{2}}-j+1
$$

When $R_{1}=R, R_{2}=R^{t}$ it reduces to the standard hook length. We have the following lemma;

\section{Lemma}

$$
\prod_{i, j \geq 1}\left(1-Q q^{h_{R_{1} R_{2}}(i, j)}\right)=\prod_{k=1}^{\infty}\left(1-Q q^{k}\right)^{k} \prod_{k}\left(1-Q q^{k}\right)^{C_{k}\left(R_{1}, R_{2}\right)}
$$

[Proof]

We first note that

$$
\begin{aligned}
\sum_{i, j \geq 1} q^{h_{R_{1} R_{2}}(i, j)} & =q\left(\sum_{i=1}^{\infty} q^{\mu_{i}^{R_{1}}-i}\right)\left(\sum_{j=1}^{\infty} q^{\mu_{j}^{R_{2}}-j}\right) \\
& =q e_{1}^{R_{1}}(q) e_{1}^{R_{2}}(q)=\widetilde{f}_{R_{1} R_{2}}(q) .
\end{aligned}
$$

Therefore

$$
\begin{aligned}
\sum_{i, j \geq 1} \log \left(1-Q q^{h_{R_{1} R_{2}}(i, j)}\right) & =-\sum_{i, j \geq 1} \sum_{n=1}^{\infty} \frac{Q^{n}}{n}\left(q^{n}\right)^{h_{R_{1} R_{2}}(i, j)}=-\sum_{n=1}^{\infty} \frac{Q^{n}}{n} \widetilde{f}_{R_{1} R_{2}}\left(q^{n}\right) \\
& =-\sum_{n=1}^{\infty} \frac{Q^{n}}{n} \frac{q^{n}}{\left(q^{n}-1\right)^{2}}-\sum_{n=1}^{\infty} \frac{Q^{n}}{n} \sum C_{k}\left(R_{1}, R_{2}\right) q^{k n} \\
& =-\sum_{n=1}^{\infty} \frac{Q^{n}}{n} \frac{q^{n}}{\left(q^{n}-1\right)^{2}}+\sum C_{k}\left(R_{1}, R_{2}\right) \log \left(1-Q q^{k}\right)(
\end{aligned}
$$


On the other hand

$$
\begin{aligned}
\sum_{k=1}^{\infty} k \log \left(1-Q q^{k}\right) & =-\sum_{k=1}^{\infty} k \sum_{n=1}^{\infty} \frac{\left(Q q^{k}\right)^{n}}{n} \\
& =-\sum_{n=1}^{\infty} \frac{Q^{n}}{n}\left(q^{n} \frac{d}{d q^{n}} \sum_{k=1}^{\infty} q^{k n}\right) \\
& =-\sum_{n=1}^{\infty} \frac{Q^{n}}{n} \frac{q^{n}}{\left(1-q^{n}\right)^{2}} .
\end{aligned}
$$

Combining (C.17) and (C.18), we obtain the lemma.

Let us now proceed to Proposition 3. Our definition of $L_{R_{1} R_{2}}\left(Q_{1}, Q_{2}\right)$ is

$$
L_{R_{1} R_{2}}\left(Q_{1}, Q_{2}\right):=\sum_{R_{1}^{\prime}, R_{2}^{\prime}} Q_{1}^{\ell_{R_{1}^{\prime}}} Q_{2}^{\ell_{R_{2}^{\prime}}} W_{R_{1} R_{1}^{\prime}} W_{R_{1}^{\prime} R_{2}^{\prime}} W_{R_{2}^{\prime} R_{2}}(-1)^{\ell_{R_{1}^{\prime}}+\ell_{R_{2}^{\prime}}} q^{-\frac{1}{2}\left(\kappa_{R_{1}^{\prime}}+\kappa_{R_{2}^{\prime}}\right)} .
$$

If we use the symmetric form (C.10) for $W_{R_{1}^{\prime} R_{2}^{\prime}}$, we find that the factors involving $\kappa_{R_{i}}$ cancel nicely and we have

$$
\begin{aligned}
L_{R_{1} R_{2}}\left(Q_{1}, Q_{2}\right)= & W_{R_{1}}(q) W_{R_{2}}(q) \sum_{R_{1}^{\prime}, R_{2}^{\prime}, R_{3}^{\prime}}\left(-Q_{1}\right)^{\ell_{R_{1}^{\prime}}}\left(-Q_{2}\right)^{\ell_{R_{2}^{\prime}}} \\
& \times s_{R_{1}^{\prime}}\left(q^{\mu^{R_{1}}+\rho}\right) s_{R_{2}^{\prime}}\left(q^{\mu^{R_{2}}+\rho}\right) s_{R_{1}^{\prime t} / R_{3}^{\prime}}\left(q^{\rho}\right) s_{R_{2}^{\prime t} / R_{3}^{\prime}}\left(q^{\rho}\right) \\
= & W_{R_{1}}(q) W_{R_{2}}(q) \sum_{R_{1}^{\prime}, R_{2}^{\prime}, R_{3}^{\prime}} s_{R_{1}^{\prime}}\left(-Q_{1} q^{\mu^{R_{1}}+\rho}\right) s_{R_{2}^{\prime}}\left(-Q_{2} q^{\mu R_{2}+\rho}\right) \\
& \times s_{R_{1}^{\prime t} / R_{3}^{\prime}}\left(q^{\rho}\right) s_{R_{2}^{\prime t} / R_{3}^{\prime}}\left(q^{\rho}\right) .
\end{aligned}
$$

Now we invoke the formulas generalizing (C.13) and (C.14) [34;

$$
\begin{aligned}
& \sum_{R} s_{R / R_{1}}(x) s_{R / R_{2}}(y)=\prod_{i, j \geq 1}\left(1-x_{i} y_{j}\right)^{-1} \sum_{Q} s_{R_{2} / Q}(x) s_{R_{1} / Q}(y), \\
& \sum_{R} s_{R / R_{1}^{t}}(x) s_{R^{t} / R_{2}}(y)=\prod_{i, j \geq 1}\left(1+x_{i} y_{j}\right) \sum_{Q} s_{R_{2}^{t} / Q}(x) s_{R_{1} / Q^{t}}(y) .
\end{aligned}
$$

Note that $R_{1}$ and $R_{2}$ are exchanged in the right hand side. If we apply (C.22) for summations over $R_{1}^{\prime}$ and $R_{2}^{\prime}$ in (C.20), only the trivial representation survives in the sum of the right hand side of (C.22). Thus we find

$$
\begin{gathered}
L_{R_{1} R_{2}}\left(Q_{1}, Q_{2}\right)=W_{R_{1}}(q) W_{R_{2}}(q) \prod_{i, j \geq 1}\left(1-Q_{1} q^{\mu_{i}^{R_{1}}-i-j+1}\right)\left(1-Q_{2} q^{\mu_{i}^{R_{2}}-i-j+1}\right) \\
\times \sum_{R_{3}^{\prime}} s_{R_{3}^{\prime t}}\left(-Q_{1} q^{\mu^{R_{1}}+\rho}\right) s_{R_{3}^{\prime \prime}}\left(-Q_{2} q^{\mu^{R_{2}}+\rho}\right)
\end{gathered}
$$


Using the formula (C.14) again, we obtain

$$
\begin{aligned}
L_{R_{1} R_{2}}\left(Q_{1}, Q_{2}\right)= & W_{R_{1}}(q) W_{R_{2}}(q) \prod_{i, j \geq 1} \frac{\left(1-Q_{1} q^{\mu_{i}^{R_{1}}-i-j+1}\right)\left(1-Q_{2} q^{\mu_{i}^{R_{2}}-i-j+1}\right)}{\left(1-Q_{1} Q_{2} q^{\mu_{i}^{R_{1}}-i+\mu_{j}^{R_{2}}-j+1}\right)} \\
= & W_{R_{1}}(q) W_{R_{2}}(q) \prod_{k=1}^{\infty} \frac{\left(1-Q_{1} q^{k}\right)^{k}\left(1-Q_{2} q^{k}\right)^{k}}{\left(1-Q_{1} Q_{2} q^{k}\right)^{k}} \\
& \times \prod_{k} \frac{\left(1-Q_{1} q^{k}\right)^{C_{k}\left(R_{1}\right)}\left(1-Q_{2} q^{k}\right)^{C_{k}\left(R_{2}\right)}}{\left(1-Q_{1} Q_{2} q^{k}\right)^{C_{k}\left(R_{1}, R_{2}\right)}} .
\end{aligned}
$$

This completes our proof of Proposition 3.

\section{Appendix D : From GV invariants to SW prepotential}

Let us summarize how one can obtain the SW prepotential of 4D $S U(2)$ pure Yang-Mills theory from the GV invariants of local Hirzebruch surface.

At genus $g$ topological string amplitude that wraps the base $\mathbf{P}^{1} k$ times has the following singularity structure [4];

$$
f_{g}^{(k)}(Q)=\frac{P_{g}^{(k)}(Q)}{(1-Q)^{2 g+4 k-2}},
$$

where $Q:=Q_{F}=e^{-t_{F}}$ is the Kähler moduli of the fiber $\mathbf{P}^{1}$ and the Taylor expansion of $f_{g}^{(k)}(Q)$ gives the $\mathrm{GV}$ invariants. $P_{g}^{(k)}(Q)$ is regular at $Q=1$.

The limit which reproduces four dimensional Seiberg-Witten theory is as follows;

$$
Q_{B}=(R \Lambda)^{4}, \quad Q_{F}=e^{-4 R a}, \quad q=e^{-2 R \hbar}
$$

and $R \rightarrow 0$. The terms in the topological string amplitude that survive in this limit are

$$
\Lambda^{4 k} \sum_{g=0}^{\infty} \frac{1}{\hbar^{2-2 g}} \frac{P_{g}^{(k)}(1)}{2^{2 g-2+8 k} a^{2 g-2+4 k}}=a^{2}\left(\frac{\Lambda}{a}\right)^{4 k} \sum_{g=0}^{\infty} \frac{1}{\hbar^{2-2 g}} \frac{P_{g}^{(k)}(1)}{2^{2 g-2+8 k} a^{2 g}} .
$$

Now we list the function $P_{g}^{(k)}(Q)$ for $\mathbf{F}_{m}(m=0,1,2)$ up to instanton number three; 


\section{D.1 $g=0$}

1. $\mathbf{F}_{0}$

$$
\begin{aligned}
& P_{0}^{(1)}=2, \\
& P_{0}^{(2)}=\frac{2 Q\left(3 Q^{2}+4 Q+3\right)}{(Q+1)^{2}}, \\
& P_{0}^{(3)}=\frac{2 Q\left(4 Q^{6}+23 Q^{5}+50 Q^{4}+62 Q^{3}+50 Q^{2}+23 Q+4\right)}{\left(Q^{2}+Q+1\right)^{2}} .
\end{aligned}
$$

2. $\mathbf{F}_{1}$

$$
\begin{aligned}
& P_{0}^{(1)}=-(Q+1) \\
& P_{0}^{(2)}=\frac{2 Q^{2}\left(3 Q^{2}+4 Q+3\right)}{(Q+1)^{2}} \\
& P_{0}^{(3)}=\frac{-Q^{3}\left(27 Q^{5}+70 Q^{4}+119 Q^{3}+119 Q^{2}+70 Q+27\right)}{\left(Q^{2}+Q+1\right)^{2}} .
\end{aligned}
$$

3. $\mathbf{F}_{2}$

$$
\begin{aligned}
& P_{0}^{(1)}=Q^{2}+1 \\
& P_{0}^{(2)}=\frac{2 Q^{3}\left(3 Q^{2}+4 Q+3\right)}{(Q+1)^{2}}, \\
& P_{0}^{(3)}=\frac{2 Q^{4}\left(4 Q^{6}+23 Q^{5}+50 Q^{4}+62 Q^{3}+50 Q^{2}+23 Q+4\right)}{\left(Q^{2}+Q+1\right)^{2}} .
\end{aligned}
$$

Up to sign flip at odd instanton numbers of $\mathbf{F}_{1}$, we obtain an universal results;

$$
P_{0}^{(1)}(Q=1)=2, \quad P_{0}^{(2)}(Q=1)=5, \quad P_{0}^{(3)}(Q=1)=48,
$$

which give the coefficients of genus zero SW prepotential

$$
\mathcal{F}_{1}=\frac{1}{2^{5}}, \quad \mathcal{F}_{2}=\frac{5}{2^{14}}, \quad \mathcal{F}_{3}=\frac{3}{2^{18}} .
$$

\section{D.2 $g=1$}

1. $\mathbf{F}_{0}$

$$
P_{1}^{(1)}=0,
$$




$$
\begin{aligned}
& P_{1}^{(2)}=\frac{Q^{2}\left(9 Q^{2}+14 Q+9\right)}{(Q+1)^{2}}, \\
& P_{1}^{(3)}=\frac{4 Q^{2}\left(17 Q^{6}+84 Q^{5}+173 Q^{4}+220 Q^{3}+173 Q^{2}+84 Q+17\right)}{\left(Q^{2}+Q+1\right)^{2}} .
\end{aligned}
$$

2. $\mathbf{F}_{1}$

$$
\begin{aligned}
& P_{1}^{(1)}=0 \\
& P_{1}^{(2)}=\frac{Q^{3}\left(9 Q^{2}+14 Q+9\right)}{(Q+1)^{2}}, \\
& P_{1}^{(3)}=\frac{-2 Q^{3}\left(5 Q^{7}+94 Q^{6}+254 Q^{5}+415 Q^{4}+415 Q^{3}+254 Q^{2}+94 Q+5\right)}{\left(Q^{2}+Q+1\right)^{2}} .
\end{aligned}
$$

3. $\mathbf{F}_{2}$

$$
\begin{aligned}
& P_{1}^{(1)}=0, \\
& P_{1}^{(2)}=\frac{Q^{4}\left(9 Q^{2}+14 Q+9\right)}{(Q+1)^{2}}, \\
& P_{1}^{(3)}=\frac{4 Q^{5}\left(17 Q^{6}+84 Q^{5}+173 Q^{4}+220 Q^{3}+173 Q^{2}+84 Q+17\right)}{\left(Q^{2}+Q+1\right)^{2}} .
\end{aligned}
$$

Again we observe a sign flip at odd instanton numbers of $\mathbf{F}_{1}$. we find an universal value;

$$
P_{1}^{(1)}(Q=1)=0, \quad P_{1}^{(2)}(Q=1)=8, \quad P_{1}^{(3)}(Q=1)=3072 / 9=2^{10} / 3
$$

and the coefficients of genus one SW prepotential

$$
\mathcal{F}_{1}=0, \quad \mathcal{F}_{2}=\frac{1}{2^{13}}, \quad \mathcal{F}_{3}=\frac{1}{3 \cdot 2^{14}} .
$$

\section{D.3 $g=2,3$}

We have computed $P_{g}^{(k)}$ for $\mathbf{F}_{0}$ and found a precise agreement with the results reported in $[28,21$

1. $g=2$

$$
P_{2}^{(1)}=0
$$




$$
\begin{aligned}
& P_{2}^{(2)}=\frac{4 Q^{3}\left(3 Q^{2}+5 Q+3\right)}{(Q+1)^{2}}, \\
& P_{2}^{(3)}=\frac{2 Q^{2}\left(6 Q^{8}+218 Q^{7}+937 Q^{6}+1868 Q^{5}+2366 Q^{4}+1868 Q^{3}+937 Q^{2}+218 Q+6\right)}{\left(Q^{2}+Q+1\right)^{2}} .
\end{aligned}
$$

2. $g=3$

$$
\begin{aligned}
& P_{3}^{(1)}=0, \\
& P_{3}^{(2)}=\frac{Q^{4}\left(15 Q^{2}+26 Q+15\right)}{(Q+1)^{2}}, \\
& P_{3}^{(3)}=\frac{4 Q^{3}\left(39 Q^{8}+624 Q^{7}+2379 Q^{6}+4616 Q^{5}+5780 Q^{4}+4616 Q^{3}+2379 Q^{2}+624 Q+39\right)}{\left(Q^{2}+Q+1\right)^{2}} .
\end{aligned}
$$

We find

$$
P_{2}^{(1)}(Q=1)=0, \quad P_{2}^{(2)}(Q=1)=11, \quad P_{2}^{(3)}(Q=1)=16848 / 9=2^{4} \cdot 3^{2} \cdot 13
$$

and

$$
\mathcal{F}_{1}=0, \quad \mathcal{F}_{2}=\frac{11}{2^{18}}, \quad \mathcal{F}_{3}=\frac{3^{2} \cdot 13}{2^{22}}
$$

for genus two prepotential and for genus three

$$
P_{3}^{(1)}(Q=1)=0, \quad P_{3}^{(2)}(Q=1)=14, \quad P_{3}^{(3)}(Q=1)=84384 / 9=2^{5} \cdot 293,
$$

and

$$
\mathcal{F}_{1}=0, \quad \mathcal{F}_{2}=\frac{7}{2^{19}}, \quad \mathcal{F}_{3}=\frac{293}{2^{23}}
$$

\section{References}

[1] M. Aganagic, M. Mariño and C. Vafa, All Loop Topological String Amplitudes From Chern-Simons Theory, hep-th/0206164.

[2] D.E. Diaconescu, B. Florea and A. Grassi, Geometric Transitions and Open String Instantons, hep-th/0205234 Geometric Transitions, del Pezzo surfaces and Open String Instantons, hep-th/0206163 
[3] A. Iqbal, All Genus Topological Amplitudes and 5-brane Webs as Feynman Diagrams, hep-th/0207114.

[4] A. Iqbal and A.-K. Kashani-Poor, Instanton Counting and Chern-Simons Theory, hep-th/0212279.

[5] A. Iqbal and A.-K. Kashani-Poor, $S U(N)$ Geometries and Topological String Amplitudes, hep-th/0306032.

[6] D.E. Diaconescu and B. Florea, Localization and Gluing of Topological Amplitudes, hep-th/0309143.

[7] R. Gopakumar and C. Vafa, M theory and Topological Strings II, hep-th/9812127.

[8] N. Nekrasov, Seiberg-Witten Prepotential from Instanton Counting, hep-th/0206161.

[9] N. Seiberg and E. Witten, Electric-Magnetic Duality, Monopole Condensation, and Confinement in $N=2$ Supersymmetric Yang-Mills Theory, Nucl. Phys. B426 (1994) 19, hep-th/9407087; Monopole, Duality, and Chiral Symmetry Breaking in $N=2$ Supersymmetric QCD, Nucl. Phys. B431 (1994) 484, hep-th/9408099.

[10] A. Losev, A. Marshakov and N. Nekrasov, Small Instantons, Little Strings and Free Fermions, hep-th/0302191.

[11] N. Nekrasov and A. Okounkov, Seiberg-Witten Prepotential and Random Partitions, hep-th/0306238.

[12] H. Nakajima and K. Yoshioka, Instanton Counting on Blowup I, math.AG/0306198.

[13] N. Nekrasov, Five-dimensional Gauge Theories and Relativistic Integrable Systems, Nucl. Phys. B 531 (1998) 323, hep-th/9609219.

[14] A. Lawrence and N. Nekrasov, Instanton Sums and Five-dimensional Gauge Theories, Nucl. Phys. B 513 (1998) 239, hep-th/9706025. 
[15] H. Kanno and Y. Ohta, Picard-Fuchs Equation and Prepotential of Five-dimensional SUSY Gauge Theory Compactified on a Circle, Nucl. Phys. B 530 (1998) 73, hep-th/9801036.

[16] T. Eguchi and H. Kanno, Five-dimensional Gauge Theories and Local Mirror Symmetry, Nucl. Phys. B 586 (2000) 331, hep-th/0005008.

[17] M. Aganagic, A. Klemm, M. Mariño and C. Vafa, The Topological Vertex, hep-th/0305132.

[18] S. Katz, A. Klemm and C. Vafa, Geometric Engineering of Quantum Field Theory, Nucl. Phys. B 497 (1997) 173, hep-th/9609239.

[19] S. Katz, P. Mayr and C. Vafa, Mirror Symmetry and Exact Solution of 4D $N=2$ Gauge Theories I, Adv. Theor. Math. Phys. 1 (1998) 53, hep-th/9706110.

[20] S. Katz and C. Vafa, Matter from Geometry, Nucl. Phys. B497 (1997) 146, hep-th/9606086.

[21] S. Hosono, Counting BPS States via Holomorphic Anomaly Equations, hep-th/0206206.

[22] M. Jinzenji and M. Naka, private communication 2001.

[23] T.-M. Chiang, A. Klemm, S.-T. Yau and E. Zaslow, Local Mirror Symmetry: Calculation and Interpretation, Adv. Theor. Math. Phys. 3 (1999) 495, hep-th/9903053.

[24] N. Seiberg, Five-dimensional SUSY field theories, non-trivial fixed points and string dynamics, Phys. Lett. B388 (1996) 753, hep-th/9608111.

[25] E. D'Hoker, I.M. Krichever and D.H. Phong, The Effective Prepotential of N=2 Supersymmetric $S U\left(N_{c}\right)$ Gauge Theories, Nucl. Phys. B489 (1997) 179, hep-th/9609041. 
[26] Y. Ohta, Prepotential of $N=2 S U(2)$ Yang-Mills Gauge Theory Coupled with a Massive Matter Multiplet, J. Math. Phys. 37 (1996) 6074, hep-th/9604051.

[27] M. Bershadsky, S. Ceccoti, H. Ooguri and C. Vafa, Kodaira-Spencer Theory of Gravity and Exact Results of Quantum String Amplitudes, Commun. Math. Phys. 165 (1994) 311, hep-th/9309140.

[28] A. Klemm, M.Mariño and S. Theisen, Gravitational Corrections in Supersymmetric Gauge Theory and Matrix Models, hep-th/0211216.

[29] R. Dijkgraaf, A. Sinkovics and M. Temürhan, Matrix Models and Gravitational Corrections, hep-th/0211241.

[30] G. Moore and E. Witten, Integration over the $u$-plane in Donaldson Theory, Adv. Theor. Math. Phys. 1 (1998) 298, hep-th/9709193.

[31] E. Witten, Quantum Field Theory and the Jones Polynomial, Commun. Math. Phys. 121 (1989) 351.

[32] H.R Morton and S.G Lukac, The HOMFLY polynomial of the Decorated Hopf Link, math.GT/0108011.

[33] A. Okounkov, N. Reshtihkin and C. Vafa, Quantum Calabi-Yau and Classical Crystals, hep-th/0309208.

[34] I.G. Macdonald, Symmetric functions and Hall polynomials, Second Edition (1995), Oxford.

[35] T. Hollowood, A. Iqbal and C. Vafa, Matrix Models, Geometric Engineering and Elliptic Genera, hep-th/0310272.

[36] J. Zhou, Localizations on Moduli Spaces and Free Field Realizations of Feynman Rules, math.AG/0310283. 